\title{
Generalized teleparallel theory
}

\author{
Ednaldo L. B. Junior ${ }^{1,2, a}$, Manuel E. Rodrigues ${ }^{1,3, b}$ \\ ${ }^{1}$ Faculdade de Física, PPGF, Universidade Federal do Pará, Belém, Pará 66075-110, Brazil \\ 2 Faculdade de Engenharia da Computação, Universidade Federal do Pará, Campus Universitrio de Tucuruí, Tucuruí, \\ Pará CEP: 68464-000, Brazil \\ ${ }^{3}$ Faculdade de Ciências Exatas e Tecnologia, Universidade Federal do Pará, Campus Universitário de Abaetetuba, Abaetetuba, \\ Pará CEP: 68440-000, Brazil
}

Received: 6 November 2015 / Accepted: 22 June 2016 / Published online: 6 July 2016 (C) The Author(s) 2016. This article is published with open access at Springerlink.com

\begin{abstract}
We construct a theory in which the gravitational interaction is described only by torsion, but that generalizes the teleparallel theory still keeping the invariance of local Lorentz transformations in one particular case. We show that our theory falls, in a certain limit of a real parameter, under $f(\bar{R})$ gravity or, in another limit of the same real parameter, under modified $f(T)$ gravity; on interpolating between these two theories it still can fall under several other theories. We explicitly show the equivalence with $f(\bar{R})$ gravity for the cases of a Friedmann-Lemaître-Robertson-Walker flat metric for diagonal tetrads, and a metric with spherical symmetry for diagonal and non-diagonal tetrads. We study four applications, one in the reconstruction of the de Sitter universe cosmological model, for obtaining a static spherically symmetric solution of de Sitter type for a perfect fluid, for evolution of the state parameter $\omega_{\mathrm{DE}}$, and for the thermodynamics of the apparent horizon.
\end{abstract}

\section{Introduction}

One of the most important findings in modern physics is that our universe has accelerated expansion [1-3]. However, a plausible common explanation for this is using the model of a very exotic fluid called dark energy, which has negative pressure. Another well-known possibility is to modify Einstein's general relativity (GR) [4], making the action of the theory depend on a function of the curvature scalar $R$, but at a certain limit of parameters the theory falls under GR. This way to explain the accelerated expansion of our universe is known as modified or generalized gravity. Considering that the gravitational interaction is described only by the curvature of space-time, we can generalize the Einstein-

\footnotetext{
${ }^{a}$ e-mail: ednaldobarrosjr@gmail.com

be-mail: esialg@gmail.com
}

Hilbert action through an analytic function of scalars of the theory, as for example $f(\bar{R})$ gravities [5-9], with $\bar{R}$ being the Ricci scalar or curvature scalar, $f(\bar{R}, \Theta)$ [10-13], with $\Theta$ being the trace of energy-momentum tensor, or yet $f(G)$ [14-18], $f(\bar{R}, G)$ [19-24] and $f\left(\bar{R}, \Theta, \bar{R}_{\mu \nu} \Theta^{\mu \nu}\right)$ [25], with $\Theta^{\mu v}$ being the energy-momentum tensor.

An alternative to consistently describe the gravitational interaction is one which only considers the torsion of spacetime, thus canceling out any effect of the curvature. This approach is known as teleparallel theory (TT) [26-29], which is demonstrably equivalent to GR. In order to describe not only the gravitational interaction, but also the accelerated expansion of our universe, Ferraro and Fiorini [30] proposed a possible generalization of the TT, which became known as $f(T)$ gravity [31-62], which up to now has provided good results in both cosmology and local phenomena of gravitation. A key problem in $f(T)$ gravity is that it breaks the invariance under local Lorentz transformations complicating the interpretation of the relationship between all inertial frames of the tangent space to the differentiable manifold (space-time) [63,64]. This problem may lead to the emergence of spurious new degrees of freedom, which are responsible for the breakdown of the local Lorentz symmetry [65]. A consequence of the formulation of the theory using a scalar which is not invariant under local Lorentz transformations, the torsion scalar $T$ in this case, is that instead of the theory presenting differential equations of motion of fourth order, as in the case of $f(\bar{R})$ gravity, it has second-order differential equations. That seems like a benefit but it is a consequence of the fact of the local Lorentz symmetry. This generalization of the TT still is not equivalent to a generalization $f(\bar{R})$ for RG.

This is the main reason why we will address the construction of a theory that generalizes the TT, but which still keeps the local Lorentz symmetry in a particular case. Therefore, it is clear that we must build the function of the action with 
dependence on a scalar that at some limit is invariant under local Lorentz transformations. This will be shown soon.

The paper is organized as follows. In Sect. 2 we present a review of $f(T)$ gravity, introducing the functional variation method used in this work, obtaining the equations of motion of this theory, noting a poorly treated point at the limit to GR. In Sect. 3 we propose the action of generalized teleparallel theory, we obtain the equations of motion through functional variation of the same and compare with $f(T)$ gravity. We show the equivalence of our theory with $f(\bar{R})$ gravity, in the case of cosmology for the line element of flat FLRW metric in Sect. 4.1, and also in the case of a spherically symmetric line element in Sect. 4.2. We also show the equivalence of our theory with a particular case of $f(T, B)$ gravity in Sect. 5 . In Sect. 6 we make four applications, one where we reconstructed the action of our theory for the universe of the model of de Sitter, and another where we obtain a static de Sitter type solution; we analyze the evolution for the state parameter to dark energy and the thermodynamics for a cosmological model. We present our final considerations in Sect. 7.

\section{The equations of motion for $f(T)$ gravity}

The geometry of a space-time can be characterized by the curvature and torsion. In the particular case in which we only consider the curvature and torsion being zero, we have defined, together with the metricity condition $\nabla_{\mu} g_{\alpha \beta} \equiv 0$ where $g_{\alpha \beta}$ are the components of the metric tensor, a Riemannian geometry where the connection $\bar{\Gamma}_{\alpha \beta}^{\mu}$ is symmetric in the last two indices. Already in the particular case that we consider only torsion (Riemann tensor identically zero, the case without curvature) in the space-time, we can then work with objects that depend solely on the so-called tetrad matrices and their derivatives as dynamic fields.

In the space-time having only torsion, the line element can be represented through two standard forms

$\mathrm{d} S^{2}=g_{\mu \nu} \mathrm{d} x^{\mu} \mathrm{d} x^{\nu}=\eta_{a b} \theta^{a} \theta^{b}$,

where we have the following relationships: $g_{\mu \nu}=\eta_{a b} e_{\mu}^{a} e_{v}^{b}$, $g^{\mu \nu}=\eta^{a b} e_{a}^{\mu} e_{b}^{v}, \theta^{a}=e_{\mu}^{a} \mathrm{~d} x^{\mu}, e_{\mu}^{a} e_{a}^{\nu}=\delta_{\mu}^{v}$ e $e_{\mu}^{a} e_{b}^{\mu}=\delta_{b}^{a}$, with $e_{\mu}^{a}$ being the tetrad matrices and $e_{a}^{\mu}$ their inverse, and $\left[\eta_{a b}\right]=$ $\operatorname{diag}[1,-1,-1,-1]$ the Minkowski metric. We adopt Latin indices for the tangent space and Greeks for space-time.

We will first establish the equations of motion for the theory $f(T)$, thus showing that the functional variation method adopted here is consistent.

We restrict the geometry to Weitzenböck geometry where we have the following connection:

$\Gamma_{\mu \nu}^{\sigma}=e_{a}^{\sigma} \partial_{\nu} e_{\mu}^{a}=-e_{\mu}^{a} \partial_{\nu} e_{a}^{\sigma}$
All Riemann tensor components are identically zero for the connection (2). We can then define the components of the tensor of torsion and contortion as

$T_{\mu \nu}^{\sigma}=\Gamma_{\nu \mu}^{\sigma}-\Gamma_{\mu \nu}^{\sigma}=e_{a}^{\sigma}\left(\partial_{\mu} e_{\nu}^{a}-\partial_{\nu} e_{\mu}^{a}\right)$,

$K_{\alpha}^{\mu \nu}=-\frac{1}{2}\left(T_{\alpha}^{\mu \nu}-T_{\alpha}^{v \mu}-T_{\alpha}^{\mu \nu}\right)$.

We can also define a new tensor, so we write the equations of motion in a more elegant way, through the components of the tensor torsion and contortion, as

$S_{\alpha}^{\mu \nu}=\frac{1}{2}\left(K_{\alpha}^{\mu \nu}+\delta_{\alpha}^{\mu} T_{\beta}^{\beta \nu}-\delta_{\alpha}^{v} T_{\beta}^{\beta \mu}\right)$.

We define the torsion scalar as

$T=T_{\mu \nu}^{\alpha} S_{\alpha}^{\mu \nu}=\frac{1}{4} T_{\mu \nu}^{\alpha} T_{\alpha}^{\mu \nu}+\frac{1}{2} T_{\mu \nu}^{\alpha} T_{\alpha}^{\nu \mu}-T_{\mu \alpha}^{\alpha} T_{\beta}^{\beta \mu}$.

Some observations are important here. The first is that there is a direct analogy to a space only with torsion and another considering only curvature in that the connections are related by

$\bar{\Gamma}_{\mu \nu}^{\alpha}=\Gamma_{\mu \nu}^{\alpha}-g_{\mu \lambda} K_{v}^{\alpha \lambda}$,

where $\bar{\Gamma}_{\mu \nu}^{\alpha}$ is the Levi-Civita connection, which is symmetric in the last two indices. The second observation is that the torsion scalar $T$ is not a Lorentz scalar (in the tangent space), being only a scalar in the tensorial indices (space-time) [66]. This is precisely the cause for that theory, being built starting from this scalar, to break down the invariance by local Lorentz transformations. We can in reality build the curvature scalar analog, through the torsion scalar, by the relation [66]

$\bar{R}=-T-2 \bar{\nabla}^{\mu} T_{\mu \alpha}^{\alpha}=-T-2 e^{-1} \partial_{\mu}\left(e g^{\mu \lambda} T_{\lambda \alpha}^{\alpha}\right)$,

where $e=\operatorname{det}\left[e_{\mu}^{a}\right]=\sqrt{-g}$, with $g=\operatorname{det}\left[g_{\mu \nu}\right]$. The curvature scalar $\bar{R}$ in (8) is a Lorentz scalar as well as a scalar on tensorial indices. That is why $f(\bar{R})$ gravity is a theory that is invariant under local Lorentz transformations and general coordinates transformations (tensorial).

It is then possible to construct a generalization of the teleparallel theory (TT) using the following action of the $f(T)$ gravity:

$S_{f(T)}=\int \mathrm{d}^{4} x \mathcal{L}_{f(T)}=\int \mathrm{d}^{4} x\left[\frac{e}{2 \kappa^{2}} f(T)-\mathcal{L}_{\text {matter }}\right]$

where $\kappa^{2}=8 \pi G_{\text {Newton }}, f(T)$ is a function of the torsion scalar and $\mathcal{L}_{\text {matter }}$ is the Lagrangian density of the matter content. We call attention to the true sign $(-)$ in front of the matter term. This so far has not been explicitly addressed in the literature of this theory, because we still have few models that couple the matter contents that need to be obtained through functional variation in principle. This feature is essential if the theory is to be equivalent to GR at some limit. It will soon be made clear. 
Addressing the functional variation of the action (9) we have

$$
\begin{aligned}
\delta S_{f(T)}= & \frac{1}{2 \kappa^{2}} \int \mathrm{d}^{4} x\left[f \delta e+e \delta f-2 \kappa^{2} \delta \mathcal{L}_{\text {matter }}\right], \\
= & \frac{1}{2 \kappa^{2}} \int \mathrm{d}^{4} x\left[f \frac{\partial e}{\partial e_{\sigma}^{a}} \delta e_{\sigma}^{a}+e \frac{\mathrm{d} f}{\mathrm{~d} T} \delta T\right] \\
& -\int \mathrm{d}^{4} x \delta \mathcal{L}_{\text {matter }}, \\
= & \delta S_{T}-\delta S_{\text {matter }},
\end{aligned}
$$

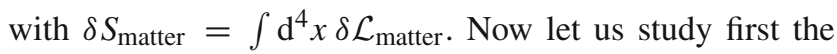
functional variation of the matter term,

$\delta S_{\text {matter }}=\int \mathrm{d}^{4} x\left[\frac{\partial \mathcal{L}_{\text {matter }}}{\partial e_{\sigma}^{a}} \delta e_{\sigma}^{a}+\frac{\partial \mathcal{L}_{\text {matter }}}{\partial\left(\partial_{\alpha} e_{\sigma}^{a}\right)} \delta\left(\partial_{\alpha} e_{\sigma}^{a}\right)\right] ;$

performing the integration by parts of the latter term, considering $\left.\delta e_{\sigma}^{a}\right|_{\text {surface }} \equiv 0$, we have

$$
\begin{aligned}
\delta S_{\text {matter }}= & \frac{1}{2 \kappa^{2}} \int \mathrm{d}^{4} \times 2 \kappa^{2}\left[\frac{\partial \mathcal{L}_{\text {matter }}}{\partial e_{\sigma}^{a}} \delta e_{\sigma}^{a}\right. \\
& \left.-\partial_{\alpha}\left(\frac{\partial \mathcal{L}_{\text {matter }}}{\partial\left(\partial_{\alpha} e_{\sigma}^{a}\right)}\right) \delta e_{\sigma}^{a}\right] \\
= & \frac{1}{2 \kappa^{2}} \int \mathrm{d}^{4} \times 2 \kappa^{2} e \Theta_{a}^{\sigma} \delta e_{\sigma}^{a}
\end{aligned}
$$

where $\Theta_{a}^{\sigma}=e_{a}^{\beta} \Theta_{\beta}^{\sigma}$, and we define $\Theta_{v}^{\sigma}$ as the energymomentum tensor.

We now have the functional variation of geometric part,

$$
\begin{aligned}
\delta S_{T}= & \frac{1}{2 \kappa^{2}} \int \mathrm{d}^{4} x\left\{f \frac{\partial e}{\partial e_{\sigma}^{a}} \delta e_{\sigma}^{a}\right. \\
& \left.+e \frac{\mathrm{d} f}{\mathrm{~d} T}\left[\frac{\partial T}{\partial e_{\sigma}^{a}} \delta e_{\sigma}^{a}+\frac{\partial T}{\partial\left(\partial_{\alpha} e_{\sigma}^{a}\right)} \delta\left(\partial_{\alpha} e_{\sigma}^{a}\right)\right]\right\} .
\end{aligned}
$$

for Performing integration by parts the last term, considering $\left.\delta e_{\sigma}^{a}\right|_{\text {surface }} \equiv 0$, we obtain

$$
\begin{aligned}
\delta S_{T}= & \frac{1}{2 \kappa^{2}} \int \mathrm{d}^{4} x\left\{f \frac{\partial e}{\partial e_{\sigma}^{a}}+e f_{T} \frac{\partial T}{\partial e_{\sigma}^{a}}\right. \\
& \left.-\partial_{\alpha}\left[e f_{T} \frac{\partial T}{\partial\left(\partial_{\alpha} e_{\sigma}^{a}\right)}\right]\right\} \delta e_{\sigma}^{a},
\end{aligned}
$$

where $f_{T}=\mathrm{d} f / \mathrm{d} T$. Taking (11) and (12) and replacing in (10), and imposing the principle of least action $\delta S_{f(T)} \equiv 0$ and multiplying by $e^{-1} e_{v}^{a} / 2$, we have the following equation of motion:

$$
\begin{aligned}
& \frac{1}{2} f\left(e^{-1} e_{\nu}^{a} \frac{\partial e}{\partial e_{\sigma}^{a}}\right)+\frac{1}{2} f_{T} e_{\nu}^{a} \frac{\partial T}{\partial e_{\sigma}^{a}} \\
& \quad-\frac{1}{2} e^{-1} e_{\nu}^{a} \partial_{\alpha}\left[e f_{T} \frac{\partial T}{\partial\left(\partial_{\alpha} e_{\sigma}^{a}\right)}\right]-\kappa^{2} \Theta_{\nu}^{\sigma}=0 .
\end{aligned}
$$

Substituting the derivatives [66]

$$
\frac{\partial e}{\partial e_{\sigma}^{a}}=e e_{a}^{\sigma}, \frac{\partial T}{\partial e_{\sigma}^{a}}=-4 e_{a}^{\lambda} T_{\nu \lambda}^{\alpha} S_{\alpha}^{\nu \sigma}, \frac{\partial T}{\partial\left(\partial_{\alpha} e_{\sigma}^{a}\right)}=4 e_{a}^{\lambda} S_{\lambda}^{\alpha \sigma},
$$

in (13) we finally have the equations of motion of $f(T)$ gravity

$$
\frac{1}{2} f \delta_{v}^{\sigma}-2 f_{T} T_{\beta \nu}^{\alpha} S_{\alpha}^{\beta \sigma}-2 e^{-1} e_{\nu}^{a} \partial_{\alpha}\left[e f_{T} e_{a}^{\beta} S_{\beta}^{\alpha \sigma}\right]-\kappa^{2} \Theta_{\nu}^{\sigma}=0
$$

Now we make use of the identity [66]

$$
\left[e^{-1} e_{\nu}^{a} \partial_{\alpha}\left(e e_{a}^{\beta} S_{\beta}^{\alpha \sigma}\right)+T_{\beta \nu}^{\alpha} S_{\alpha}^{\beta \sigma}\right]=-\frac{1}{2}\left[G_{v}^{\sigma}-\frac{1}{2} \delta_{\nu}^{\sigma} T\right],
$$

with $G_{v}^{\sigma}$ being the mixed components of the Einstein tensor, for rewriting (15) as

$$
-2 S_{v}^{\alpha \sigma} \partial_{\alpha} f_{T}+f_{T} G_{v}^{\mu}+\frac{1}{2} \delta_{v}^{\sigma}\left[f-f_{T} T\right]=\kappa^{2} \Theta_{v}^{\sigma} .
$$

This theory falls under Einstein's general relativity with a cosmological constant, when we make $f(T)=T-2 \Lambda$. Here it becomes clear that if we do not consider the sign $(-)$ in front of the matter term in the action (9) in the theory, we do not return to GR for a linear $f(T)$ function, reaching the case opposite to Einstein's equation. This fact will be crucial in showing later that an invariant theory by local Lorentz transformations, as $f(\bar{R})$ gravity, cannot fall under $f(T)$ gravity, since these have opposite coupling signs to the matter term.

Sotiriou et al. $[63,64]$ have shown that $f(T)$ gravity does not preserve its equations of motion invariant by local Lorentz transformations. It is in relation to this problem that we then construct a generalization of the teleparallel theory that preserves the invariance of the equations of motion for a local Lorentz transformation. This will be addressed in the next section.

\section{Equations of motion on generalized teleparallel theory}

An important identity is given by $\bar{R}=-T-2 \bar{\nabla}^{\mu} T_{\mu \beta}^{\beta}$, where $\bar{R}$ is the curvature scalar associated with a Riemann tensor defined solely by the Levi-Civita connection $\bar{\Gamma}_{\mu \nu}^{\alpha}$, where the indices $(\mu \nu)$ are symmetric, and the covariant derivative $\bar{\nabla}$ is defined by this connection. The curvature scalar is by definition invariant under a local Lorentz transformation, but it is also invariant under a general coordinate transformation. So it would be interesting to develop a theory that generalizes the TT but for which the functional action depends on an invariant under local Lorentz transformations. This is not the case of $f(T)$ gravity.

We propose the following action:

$$
S_{G T T}=\int \mathrm{d}^{4} x\left[\frac{e}{2 \kappa^{2}} f(\mathcal{T})+\mathcal{L}_{\text {matter }}\right]
$$


where we define

$$
\mathcal{T}=-T-2 a_{1} \bar{\nabla}^{\mu} T_{\mu \beta}^{\beta}=-T-2 a_{1} e^{-1} \partial_{\mu}\left(e g^{\mu \lambda} T_{\lambda \alpha}^{\alpha}\right) .
$$

This action generalizes TT and falls under a modified $f(T)$ gravity as well as $f(\bar{R})$ gravity. We can show this by making the limit $a_{1} \rightarrow 0$, where we have $\mathcal{T} \rightarrow-T$, therefore $f(\mathcal{T}) \rightarrow f(-T), f_{\mathcal{T}} \rightarrow-f_{T}$ and the theory must be equivalent to a modified $f(T)$ (we shall see this later). Moreover, we can regain $f(\bar{R})$ gravity, making the limit $a_{1} \rightarrow 1$, where we have $\mathcal{T} \rightarrow \bar{R}$; then the theory must be equivalent to $f(\bar{R})$. We show this explicitly through the equations of motion later on.

By performing the functional variation of the action (18) we obtain

$\delta S_{G T T}=\frac{1}{2 \kappa^{2}} \int \mathrm{d}^{4} x\left[f \delta e+e \delta f+2 \kappa^{2} \delta \mathcal{L}_{\text {matter }}\right]$.

As $S_{G T T} \equiv S_{G T T}\left[e_{\sigma}^{a}, \partial_{\alpha} e_{\sigma}^{a}, \Phi^{A}\right]$, in which $\Phi^{A}$ are the matter fields, we have

$\delta S_{G T T}=\delta S_{\mathcal{T}}+\delta S_{\text {matter }}$,

with $\delta S_{\text {matter }}=\int \mathrm{d}^{4} x \delta \mathcal{L}_{\text {matter }}$ in the same manner as in $f(T)$ gravity. The functional variation of the matter term (21) is exactly the same as given in (11).

The geometric part is

$$
\begin{aligned}
\delta S_{\mathcal{T}} & =\frac{1}{2 \kappa^{2}} \int \mathrm{d}^{4} x[f \delta e+e \delta f] \\
& =\frac{1}{2 \kappa^{2}} \int \mathrm{d}^{4} x\left[f \frac{\partial e}{\partial e_{\sigma}^{a}} \delta e_{\sigma}^{a}+e f_{\mathcal{T}} \delta \mathcal{T}\right],
\end{aligned}
$$

where we use $f_{\mathcal{T}}=\mathrm{d} f / \mathrm{d} \mathcal{T}$. The first term in (22) is already known, and we will pay attention to the second term.

Performing the functional variation to $\mathcal{T}$ in (19) we obtain

$$
\begin{aligned}
\delta \mathcal{T}= & -\delta T-2 a_{1} \delta\left[e^{-1} \partial_{\mu}\left(e g^{\mu \beta} T_{\beta \alpha}^{\alpha}\right)\right] \\
= & -\delta T-2 a_{1}\left[-e^{-2} \partial_{\mu}\left(e g^{\mu \beta} T_{\beta \alpha}^{\alpha}\right) \delta e\right. \\
& \left.+e^{-1} \delta \partial_{\mu}\left(e g^{\mu \beta} T_{\beta \alpha}^{\alpha}\right)\right],
\end{aligned}
$$

in (22) taking into account the functional variation of $T$ and $e$ we have

$$
\begin{aligned}
\delta S_{\mathcal{T}}= & \frac{1}{2 \kappa^{2}} \int \mathrm{d}^{4} x\left\{f \frac{\partial e}{\partial e_{\sigma}^{a}} \delta e_{\sigma}^{a}\right. \\
& -e f_{\mathcal{T}}\left[\frac{\partial T}{\partial e_{\sigma}^{a}} \delta e_{\sigma}^{a}+\frac{\partial T}{\partial\left(\partial_{\alpha} e_{\sigma}^{a}\right)} \delta\left(\partial_{\alpha} e_{\sigma}^{a}\right)\right] \\
& +2 a_{1}\left[e^{-1} f_{\mathcal{T}} \partial_{\mu}\left(e g^{\mu \beta} T_{\beta \alpha}^{\alpha}\right) \frac{\partial e}{\partial e_{\sigma}^{a}} \delta e_{\sigma}^{a}\right. \\
& \left.\left.-f_{\mathcal{T}} \delta \partial_{\mu}\left(e g^{\mu \beta} T_{\beta \nu}^{v}\right)\right]\right\} .
\end{aligned}
$$

Now we do the integration by parts in the terms containing $\delta\left(\partial_{\alpha} e_{\sigma}^{a}\right)$ and $\delta \partial_{\mu}\left(e g^{\mu \beta} T_{\beta \nu}^{v}\right)$. The first integration by parts is given by

$$
\begin{aligned}
- & \frac{1}{2 \kappa^{2}} \int \mathrm{d}^{4} x e f_{\mathcal{T}} \frac{\partial T}{\partial\left(\partial_{\alpha} e_{\sigma}^{a}\right)} \delta\left(\partial_{\alpha} e_{\sigma}^{a}\right) \\
= & -\frac{1}{2 \kappa^{2}} \int \mathrm{d}^{4} x \partial_{\alpha}\left[e f_{\mathcal{T}} \frac{\partial T}{\partial\left(\partial_{\alpha} e_{\sigma}^{a}\right)} \delta e_{\sigma}^{a}\right] \\
& +\frac{1}{2 \kappa^{2}} \int \mathrm{d}^{4} x \partial_{\alpha}\left[e f_{\mathcal{T}} \frac{\partial T}{\partial\left(\partial_{\alpha} e_{\sigma}^{a}\right)}\right] \delta e_{\sigma}^{a},
\end{aligned}
$$

where the first term is zero because it is a surface term, which we consider $\left.\delta e_{\sigma}^{a}\right|_{\text {surface }} \equiv 0$. The second integration by parts is given by

$$
\begin{aligned}
- & \frac{2 a_{1}}{2 \kappa^{2}} \int \mathrm{d}^{4} x f_{\mathcal{T}} \delta \partial_{\mu}\left(e g^{\mu \beta} T_{\beta \nu}^{v}\right) \\
= & -\frac{2 a_{1}}{2 \kappa^{2}} \int \mathrm{d}^{4} x \partial_{\mu}\left[f_{\mathcal{T}} \delta\left(e g^{\mu \beta} T_{\beta \nu}^{v}\right)\right] \\
& +\frac{2 a_{1}}{2 \kappa^{2}} \int \mathrm{d}^{4} x\left(\partial_{\mu} f_{\mathcal{T}}\right) \delta\left(e g^{\mu \beta} T_{\beta \nu}^{v}\right),
\end{aligned}
$$

with the first term is null for being a surface term. Then we have

$$
\begin{aligned}
- & \frac{2 a_{1}}{2 \kappa^{2}} \int \mathrm{d}^{4} x f_{\mathcal{T}} \delta \partial_{\mu}\left(e g^{\mu \beta} T_{\beta \nu}^{v}\right) \\
= & \frac{2 a_{1}}{2 \kappa^{2}} \int \mathrm{d}^{4} x\left(\partial_{\mu} f_{\mathcal{T}}\right)\left[g^{\mu \beta} T_{\beta \nu}^{v} \frac{\partial e}{\partial e_{\sigma}^{a}} \delta e_{\sigma}^{a}\right. \\
& \left.+e T_{\beta \nu}^{v} \delta g^{\mu \beta}+e g^{\mu \beta} \delta T_{\beta \nu}^{v}\right] .
\end{aligned}
$$

Making use of the following relationship:

$$
\begin{aligned}
\delta g^{\mu \beta} & =\delta\left(\eta^{a b} e_{a}^{\mu} e_{b}^{\beta}\right) \\
& =-\left(g^{\beta \sigma} e_{a}^{\mu} \delta e_{\sigma}^{a}+g^{\mu \sigma} e_{a}^{\beta} \delta e_{\sigma}^{a}\right)
\end{aligned}
$$

and replacing (25) and (27) in (24), developing the terms of $\delta T_{\beta \nu}^{v}$ we have

$$
\begin{aligned}
\delta S_{\mathcal{T}}= & \frac{1}{2 \kappa^{2}} \int \mathrm{d}^{4} x\left\{f \frac{\partial e}{\partial e_{\sigma}^{a}} \delta e_{\sigma}^{a}-e f_{\mathcal{T}} \frac{\partial T}{\partial e_{\sigma}^{a}} \delta e_{\sigma}^{a}\right. \\
& +\partial_{\alpha}\left[e f_{\mathcal{T}} \frac{\partial T}{\partial\left(\partial_{\alpha} e_{\sigma}^{a}\right)}\right] \delta e_{\sigma}^{a} \\
& +2 a_{1}\left[e^{-1} f_{\mathcal{T}} \partial_{\mu}\left(e g^{\mu \beta} T_{\beta \nu}^{v}\right) \frac{\partial e}{\partial e_{\sigma}^{a}} \delta e_{\sigma}^{a}\right. \\
& +\left(\partial_{\mu} f_{\mathcal{T}}\right)\left[g^{\mu \beta} T_{\beta \nu}^{v} \frac{\partial e}{\partial e_{\sigma}^{a}} \delta e_{\sigma}^{a}\right. \\
& -e T_{\beta \nu}^{v}\left(g^{\beta \sigma} e_{a}^{\mu} \delta e_{\sigma}^{a}+g^{\mu \sigma} e_{a}^{\beta} \delta e_{\sigma}^{a}\right)+e g^{\mu \beta} \frac{\partial T_{\beta \nu}^{v}}{\partial e_{\sigma}^{a}} \delta e_{\sigma}^{a} \\
& \left.\left.\left.+e g^{\mu \beta} \frac{\partial T_{\beta \nu}^{v}}{\partial\left(\partial_{\alpha} e_{\sigma}^{a}\right)} \delta\left(\partial_{\alpha} e_{\sigma}^{a}\right)\right]\right]\right\} .
\end{aligned}
$$


At this point we see that we still have to do an integration by parts in the last term, i.e.,

$$
\begin{aligned}
\frac{2 a_{1}}{2 \kappa^{2}} \int \mathrm{d}^{4} x\left(\partial_{\mu} f_{\mathcal{T}}\right) e g^{\mu \beta} \frac{\partial T_{\beta \nu}^{v}}{\partial\left(\partial_{\alpha} e_{\sigma}^{a}\right)} \delta\left(\partial_{\alpha} e_{\sigma}^{a}\right) \\
=\frac{2 a_{1}}{2 \kappa^{2}} \int \mathrm{d}^{4} x \partial_{\alpha}\left[\left(\partial_{\mu} f_{\mathcal{T}}\right) e g^{\mu \beta} \frac{\partial T_{\beta \nu}^{v}}{\partial\left(\partial_{\alpha} e_{\sigma}^{a}\right)} \delta e_{\sigma}^{a}\right] \\
\quad-\frac{2 a_{1}}{2 \kappa^{2}} \int \mathrm{d}^{4} x \partial_{\alpha}\left[\left(\partial_{\mu} f_{\mathcal{T}}\right) e g^{\mu \beta} \frac{\partial T_{\beta \nu}^{v}}{\partial\left(\partial_{\alpha} e_{\sigma}^{a}\right)}\right] \delta e_{\sigma}^{a},
\end{aligned}
$$

where once again the first term vanishes due to being a surface term. Substituting this result in (29) we obtain

$$
\begin{aligned}
& \delta S_{\mathcal{T}}=\frac{1}{2 \kappa^{2}} \int \mathrm{d}^{4} x\left\{f \frac{\partial e}{\partial e_{\sigma}^{a}}-e f_{\mathcal{T}} \frac{\partial T}{\partial e_{\sigma}^{a}}\right. \\
& \quad+\partial_{\alpha}\left[e f_{\mathcal{T}} \frac{\partial T}{\partial\left(\partial_{\alpha} e_{\sigma}^{a}\right)}\right]+2 a_{1}\left[e^{-1} f_{\mathcal{T}} \partial_{\mu}\left(e g^{\mu \beta} T_{\beta \nu}^{v}\right) \frac{\partial e}{\partial e_{\sigma}^{a}}\right. \\
& \quad+\left(\partial_{\mu} f_{\mathcal{T}}\right)\left[g^{\mu \beta} T_{\beta \nu}^{v} \frac{\partial e}{\partial e_{\sigma}^{a}}-e T_{\beta \nu}^{v}\left(g^{\beta \sigma} e_{a}^{\mu}+g^{\mu \sigma} e_{a}^{\beta}\right)\right. \\
& \left.\left.\left.\quad+e g^{\mu \beta} \frac{\partial T_{\beta \nu}^{v}}{\partial e_{\sigma}^{a}}\right]-\partial_{\alpha}\left[\left(\partial_{\mu} f_{\mathcal{T}}\right) e g^{\mu \beta} \frac{\partial T_{\beta \nu}^{v}}{\partial\left(\partial_{\alpha} e_{\sigma}^{a}\right)}\right]\right]\right\} \delta e_{\sigma}^{a} .
\end{aligned}
$$

Now we must replace the results derived from $T, e$ and $T_{\beta \nu}^{v}$ in the relation as regards the tetrads and the derivatives. Taking into account the results of $f(T)$, we have the following derivative:

$$
\begin{aligned}
& \frac{\partial e}{\partial e_{\sigma}^{a}}=e e_{a}^{\sigma}, \frac{\partial T}{\partial e_{\sigma}^{a}}=-4 e_{a}^{\lambda} T_{v \lambda}^{\alpha} S_{\alpha}^{\nu \sigma}, \\
& \frac{\partial T}{\partial\left(\partial_{\alpha} e_{\sigma}^{a}\right)}=4 e_{a}^{\lambda} S_{\lambda}^{\alpha \sigma}, \frac{\partial T_{\beta \nu}^{v}}{\partial e_{\sigma}^{a}}=-e_{a}^{\nu} T_{\beta \nu}^{\sigma}, \\
& \frac{\partial T_{\beta \nu}^{v}}{\partial\left(\partial_{\alpha} e_{\sigma}^{a}\right)}=e_{b}^{v} \delta_{a}^{b}\left(\delta_{\beta}^{\alpha} \delta_{v}^{\sigma}-\delta_{v}^{\alpha} \delta_{\beta}^{\sigma}\right) .
\end{aligned}
$$

Substituting the above derivatives in (31), making $\delta S_{G T T} \equiv$ 0 in (21) and multiplying by $\frac{1}{2} e^{-1} e_{\omega}^{a}$ we have the following equation of motion for the generalized teleparallel theory:

$$
\begin{aligned}
& \frac{1}{2} \delta_{\omega}^{\sigma} f+2 f_{\mathcal{T}} T_{\nu \omega}^{\beta} S_{\beta}^{\nu \sigma}+2 e^{-1} e_{\omega}^{a} \partial_{\alpha}\left[e f_{\mathcal{T}} e_{a}^{\beta} S_{\beta}^{\alpha \sigma}\right] \\
& \quad+a_{1}\left\{e^{-1} f_{\mathcal{T}} \delta_{\omega}^{\sigma} \partial_{\mu}\left(e g^{\mu \beta} T_{\beta \nu}^{\nu}\right)+\left(\partial_{\mu} f_{\mathcal{T}}\right)\left[\delta_{\omega}^{\sigma} g^{\mu \beta} T_{\beta \nu}^{\nu}\right.\right. \\
& \left.\quad-\left(\delta_{\omega}^{\mu} g^{\beta \sigma} T_{\beta \nu}^{v}+g^{\mu \sigma} T_{\omega \nu}^{v}\right)-g^{\mu \beta} T_{\beta \omega}^{\sigma}\right] \\
& \left.\quad-e^{-1} e_{\omega}^{a} \partial_{\alpha}\left[e\left(\partial_{\mu} f_{\mathcal{T}}\right)\left(g^{\mu \alpha} e_{a}^{\sigma}-g^{\mu \sigma} e_{a}^{\alpha}\right)\right]\right\}+\kappa^{2} \Theta_{\omega}^{\sigma}=0 .
\end{aligned}
$$

Taking the limit in which $a_{1} \rightarrow 0(\mathcal{T} \rightarrow-T, f \equiv$ $\left.f(-T), f_{\mathcal{T}} \rightarrow-f_{T}\right)$, making $T \rightarrow-T$ the equation of motion (34) does not fall exactly under the equation of motion of the $f(T)$ gravity in (15). This happens due to the fact that the relationship between the curvature scalar and the torsion scalar has a minus sign, which prevents a theory to be $f(\bar{R})$ gravity, in which the coupling with matter is positive, which would fall under a theory like $f(T)$ gravity, in which the coupling signal with the matter should be negative so that it falls under GR. In the next section we shall show the equivalence between GTT and $f(\bar{R})$ gravity.

\section{Equivalence between GTT and $f(\bar{R})$ gravity}

Let us start this section showing the equivalence of GTT with $f(\bar{R})$ gravity in the limit $a_{1} \rightarrow 1$, to general tetrads.

Let us first establish some necessary identities, as arising from the condition of the metricity,

$$
\begin{aligned}
\bar{\nabla}_{\alpha} g_{\mu \nu}=\bar{\nabla}_{\alpha} g^{\mu \nu} \equiv 0, \partial_{\alpha} g_{\mu \nu} & =\bar{\Gamma}_{\alpha \mu}^{\lambda} g_{\lambda \nu} \\
+\bar{\Gamma}_{\alpha \nu}^{\lambda} g_{\lambda \mu}, \partial_{\alpha} g^{\mu \nu} & =-\bar{\Gamma}_{\lambda \alpha}^{\mu} g^{\lambda \nu}-\bar{\Gamma}_{\lambda \alpha}^{\nu} g^{\lambda \mu}
\end{aligned}
$$

With it the identity $\partial_{\alpha} e=e g^{\mu \nu} \partial_{\alpha} g_{\mu \nu}$ becomes

$\partial_{\alpha} e=2 e \bar{\Gamma}_{\alpha \nu}^{v}$.

Now we can divide the equation of motion (34) in terms such as

$$
\begin{aligned}
T^{(1)}+ & T^{(2)}+T^{(3)}+\kappa^{2} \Theta_{\omega}^{\sigma}=0, \\
T^{(1)}= & \frac{1}{2} \delta_{\omega}^{\sigma} f+2 f_{\mathcal{T}} T_{\nu \omega}^{\beta} S_{\beta}^{\nu \sigma} \\
& +2 e^{-1} e_{\omega}^{a} \partial_{\alpha}\left[e f_{\mathcal{T}} e_{a}^{\beta} S_{\beta}^{\alpha \sigma}\right]+a_{1} e^{-1} f_{\mathcal{T}} \delta_{\omega}^{\sigma} \partial_{\mu}\left(e g^{\mu \beta} T_{\beta \nu}^{v}\right),
\end{aligned}
$$

$$
\begin{aligned}
T^{(2)}= & a_{1}\left(\partial_{\mu} f_{\mathcal{T}}\right)\left[\delta_{\omega}^{\sigma} g^{\mu \beta} T_{\beta \nu}^{v}-\delta_{\omega}^{\mu} g^{\beta \sigma} T_{\beta \nu}^{\nu}\right. \\
& \left.-g^{\mu \sigma} T_{\omega \nu}^{v}-g^{\mu \beta} T_{\beta \omega}^{\sigma}\right], \\
T^{(3)}= & -a_{1} e^{-1} e_{\omega}^{a} \partial_{\alpha}\left[e\left(\partial_{\mu} f_{\mathcal{T}}\right)\left(g^{\mu \alpha} e_{a}^{\sigma}-g^{\mu \sigma} e_{a}^{\alpha}\right)\right] .
\end{aligned}
$$

Developing the last term we have

$$
\begin{aligned}
T^{(3)}= & -a_{1} \delta_{\omega}^{\sigma} \bar{\square} f_{\mathcal{T}}+a_{1} g^{\mu \sigma} \bar{\nabla}_{\omega} \bar{\nabla}_{\mu} f_{\mathcal{T}}-a_{1} g^{\nu \sigma} \bar{\Gamma}^{\mu}{ }_{\omega \nu} \partial_{\mu} f_{\mathcal{T}} \\
& -a_{1} e^{-1} e^{a}{ }_{\omega}\left(\partial_{\mu} f_{\mathcal{T}}\right)\left[\left(g^{\mu \alpha} e_{a}{ }^{\sigma}-g^{\mu \sigma} e_{a}{ }^{\alpha}\right) \partial_{\alpha} e\right. \\
& +e\left(e_{a}{ }^{\sigma} \partial_{\alpha} g^{\mu \alpha}+g^{\mu \alpha} \partial_{\alpha} e_{a}{ }^{\sigma}\right. \\
& \left.\left.-e_{a}^{\alpha} \partial_{\alpha} g^{\mu \sigma}-g^{\mu \sigma} \partial_{\alpha} e_{a}^{\alpha}\right)\right] .
\end{aligned}
$$

Using (2), (3), (4), (7), (35), and (36) in (39) and (41) we have the sum of terms $T^{(2)}$ and $T^{(3)}$ resulting in

$T^{(2)}+T^{(3)}=-a_{1} \delta_{\omega}^{\sigma} \bar{\square} f_{\mathcal{T}}+a_{1} g^{\mu \sigma} \bar{\nabla}_{\omega} \bar{\nabla}_{\mu} f_{\mathcal{T}}$.

Now we use the identity (16) in (38), then we can rewrite the equation of motion (37), using (42), as follows:

$$
\begin{aligned}
- & f_{\mathcal{T}} G_{v}^{\mu}-a_{1}\left[\delta_{v}^{\mu} \bar{\square}-g^{\mu \alpha} \bar{\nabla}_{v} \bar{\nabla}_{\alpha}\right] f_{\mathcal{T}} \\
& +\frac{1}{2}\left[-\mathcal{T} f_{\mathcal{T}}+f\right] \delta_{v}^{\mu}+2 S_{v}^{\alpha \mu} \partial_{\alpha} f_{\mathcal{T}}+\kappa^{2} \Theta_{v}^{\mu}=0 .
\end{aligned}
$$


Considering $\mathcal{T} \equiv \mathcal{T}\left(-T-a_{1} B\right)$, with $B=2 \partial_{\mu}\left(e g^{\mu \beta} T_{\beta \nu}^{v}\right)$, we see that GTT will only be equivalent to $f(\bar{R})$ gravity in the limit $a_{1} \rightarrow 1$, so $\mathcal{T} \rightarrow \bar{R}$ and the term $2 S_{\nu}^{\alpha \mu} \partial_{\alpha} f_{\mathcal{T}}$ must be identically zero, as shown in Sect. III, subsection C, of [68]. When this term vanishes, we have exactly one theory invariant by local Lorentz transformations, which occurs only when $a_{1} \rightarrow 1$, and thus Eq. (43) becomes identical to $f(\bar{R})$ gravity, which is covariant and independent of the chosen of set of tetrads.

In the next section we will specify a set of tetrads that explicitly show the equivalence between the two theories in the limit referred to above.

\subsection{Friedmann-Lemaître-Robertson-Walker case}

In this section we explicitly show that the GTT equations of motion in (34) are exactly the same as $f(\bar{R})$ gravity for the particular limit in which $a_{1} \rightarrow 1$. We can then begin comparing the equations of motion for an easier symmetry of the metric, as the maximum symmetry for the cosmological Friedmann-Lemaitre-Robertson-Walker (FLRW) flat metric,

$\mathrm{d} S_{F L R W}^{2}=\mathrm{d} t^{2}-a^{2}(t)\left(\mathrm{d} x^{2}+\mathrm{d} y^{2}+\mathrm{d} z^{2}\right)$.

Considering the case of cosmology, with line element FLRW flat (44), for a diagonal tetrad $\left[e_{\sigma}^{a}\right]=\operatorname{diag}[1, a(t), a(t)$, $a(t)]$, we see that Eq. (34) become

$$
\begin{aligned}
\kappa^{2} \Theta_{0}^{0}= & \frac{1}{2 a^{2}}\left\{6 a_{1} a \dot{a}\left(\frac{\mathrm{d}}{\mathrm{d} t} f_{\mathcal{T}}\right)\right. \\
& \left.+\left[12\left(1-a_{1}\right)(\dot{a})^{2}-6 a_{1} a \ddot{a}\right] f_{\mathcal{T}}-f a^{2}\right\},
\end{aligned}
$$

$$
\begin{aligned}
\kappa^{2} \Theta_{1}^{1}= & \kappa^{2} \Theta_{2}^{2}=\kappa^{2} \Theta_{3}^{3} \\
= & -\frac{1}{2 a^{2}}\left\{2 a\left(a_{1} a \frac{\mathrm{d}}{\mathrm{d} t}+2 \dot{a}\right) \frac{\mathrm{d}}{\mathrm{d} t} f_{\mathcal{T}}+\left[\left(4-6 a_{1}\right) a \ddot{a}\right.\right. \\
& \left.\left.+\left(8-12 a_{1}\right)(\dot{a})^{2}\right] f_{\mathcal{T}}-f a^{2}\right\},
\end{aligned}
$$

where $\dot{a}=(\mathrm{d} / \mathrm{d} t) a$ and $\ddot{a}=\left(\mathrm{d}^{2} / \mathrm{d} t^{2}\right) a$.

We can now compare these equations with those obtained from $f(\bar{R})$ gravity, whose equations of motion are [5-9]

$\kappa^{2} \Theta_{\nu}^{\mu}=f_{\bar{R}} \bar{R}_{v}^{\mu}-\frac{1}{2} \delta_{\nu}^{\mu} f+\left(\delta_{\nu}^{\mu} \bar{\square}-g^{\mu \beta} \bar{\nabla}_{\beta} \bar{\nabla}_{\nu}\right) f_{\bar{R}}$.

Considering the flat FLRW metric (44), Eq. (47) provide us with

$\kappa^{2} \Theta_{0}^{0}=\frac{1}{2 a}\left[6 \dot{a} \frac{\mathrm{d}}{\mathrm{d} t} f_{R}-6 \ddot{a} f_{R}-a f\right]$,

$$
\begin{aligned}
\kappa^{2} \Theta_{1}^{1}= & \kappa^{2} \Theta_{2}^{2}=\kappa^{2} \Theta_{3}^{3} \\
= & -\frac{1}{2 a^{2}}\left[\left(2 a^{2} \frac{\mathrm{d}}{\mathrm{d} t}+4 a \dot{a}\right) \frac{\mathrm{d}}{\mathrm{d} t} f_{R}\right. \\
& \left.-\left(2 a \ddot{a}+4(\dot{a})^{2}\right) f_{R}-f a^{2}\right] .
\end{aligned}
$$

Subtracting (45) from (48) we have

$$
\begin{aligned}
& 0=\frac{3}{a^{2}}\left\{2\left(1-a_{1}\right) \dot{a}^{2} f_{\mathcal{T}}+a \dot{a}\left(a_{1} \frac{\mathrm{d} f_{\mathcal{T}}}{\mathrm{d} t}-\frac{\mathrm{d} f_{\bar{R}}}{\mathrm{~d} t}\right)\right. \\
& \left.+a \ddot{a}\left(f_{\bar{R}}-a_{1} f_{\mathcal{T}}\right)+\frac{a^{2}}{6}[f(\bar{R})-f(\mathcal{T})]\right\} .
\end{aligned}
$$

Subtracting (46) from (49) we obtain

$$
\begin{aligned}
0= & \frac{1}{a^{2}}\left\{a^{2}\left(a_{1} \frac{d^{2} f_{\mathcal{T}}}{\mathrm{d} t^{2}}-\frac{\mathrm{d}^{2} f_{\bar{R}}}{\mathrm{~d} t^{2}}\right)+2 a \dot{a}\left(\frac{\mathrm{d} f_{\mathcal{T}}}{\mathrm{d} t}-\frac{\mathrm{d} f_{\bar{R}}}{\mathrm{~d} t}\right)\right. \\
& +a \ddot{a}\left[\left(2-3 a_{1}\right) f_{\mathcal{T}}+f_{\bar{R}}\right]+\dot{a}^{2}\left[\left(4-6 a_{1}\right) f_{\mathcal{T}}+2 f_{\bar{R}}\right] \\
& \left.+\frac{a^{2}}{2}[f(\bar{R})-f(\mathcal{T})]\right\} .
\end{aligned}
$$

Now we clearly see that in the limit $a_{1} \rightarrow 1$ we have $\left\{\mathcal{T} \rightarrow \bar{R}, f(\mathcal{T}) \rightarrow f(\bar{R}), f_{\mathcal{T}} \rightarrow f_{\bar{R}}\right\}$, then (50) and (51) are identically null, showing the equivalence of equations of motion between GTT and $f(\bar{R})$ for this limit. The conclusion is that GTT is only invariant under local Lorentz transformations and at the same time invariant by general coordinates transformations in the limit at which $a_{1} \rightarrow 1$.

\subsection{Spherically symmetric case}

We have demonstrated in general that GTT is equivalent to $f(R)$ gravity, but in addition to explain this through a metric with specific symmetry, we want to leave the equations of motion open for further analysis of this theory.

Let us now consider the case of a spherically symmetric and static line element,

$\mathrm{d} S^{2}=e^{a(r)} \mathrm{d} t^{2}-e^{b(r)} \mathrm{d} r^{2}-r^{2}\left(\mathrm{~d} \theta^{2}+\sin ^{2} \theta \mathrm{d} \phi^{2}\right) ;$

we can choose the following diagonal tetrad: $\left[e_{\sigma}^{a}\right]=$ $\operatorname{diag}\left[e^{a(r) / 2}, e^{b(r) / 2}, r, r \sin \theta\right]$, which, taking into account (34), provides us with the following equations of motion:

$$
\begin{aligned}
\kappa^{2} \Theta_{0}^{0}= & -\frac{e^{-b}}{4 r^{2}}\left\{4 a_{1} r^{2} \frac{\mathrm{d}^{2}}{\mathrm{~d} r^{2}} f_{\mathcal{T}}+\left(8 r-2 a_{1} r^{2} b^{\prime}\right) \frac{\mathrm{d}}{\mathrm{d} r} f_{\mathcal{T}}\right. \\
& +\left[\left(a_{1} r^{2} a^{\prime}+4\left(a_{1}-1\right) r\right) b^{\prime}\right. \\
& +4\left(a_{1}-1\right) e^{b}-a_{1} r^{2}\left(2 a^{\prime \prime}+\left(a^{\prime}\right)^{2}\right) \\
& \left.\left.-4\left(2 a_{1}-1\right) r a^{\prime}-8\left(a_{1}-1\right)\right] f_{\mathcal{T}}+2 f r^{2} e^{b}\right\},
\end{aligned}
$$




$$
\begin{aligned}
\kappa^{2} \Theta_{1}^{1}= & \frac{e^{-b}}{4 r^{2}}\left\{a_{1}\left(2 r^{2} a^{\prime}+8 r\right) \frac{\mathrm{d}}{\mathrm{d} r} f_{\mathcal{T}}\right. \\
& +\left[a_{1} r\left(r a^{\prime}+4\right) b^{\prime}+4\left(a_{1}-1\right) e^{b}\right. \\
& -a_{1}\left(2 r^{2} a^{\prime \prime}+r^{2}\left(a^{\prime}\right)^{2}\right)+8\left(1-a_{1}\right) r a^{\prime} \\
& \left.\left.+8\left(1-a_{1}\right)\right] f_{\mathcal{T}}+2 f r^{2} e^{b}\right\},
\end{aligned}
$$

$$
\kappa^{2} \Theta_{2}^{1}=\frac{\left(a_{1}-1\right) \cos \theta \frac{\mathrm{d}}{\mathrm{d} r} f_{\mathcal{T}}}{r^{2} \sin \theta}=0,
$$

$$
\begin{aligned}
\kappa^{2} \Theta_{2}^{2}= & \kappa^{2} \Theta_{3}^{3}=\frac{e^{-b}}{4 r^{2}}\left\{4 a_{1} r^{2} \frac{\mathrm{d}^{2}}{\mathrm{~d} r^{2}} f_{\mathcal{T}}\right. \\
& -2 r\left(a_{1} r b^{\prime}-r a^{\prime}-2\right) \frac{\mathrm{d}}{\mathrm{d} r} f_{\mathcal{T}} \\
& +\left[\left(\left(a_{1}-1\right) r^{2} a^{\prime}+\left(4 a_{1}-2\right) r\right) b^{\prime}+4 a_{1} e^{b}\right. \\
& +2\left(1-a_{1}\right) r^{2} a^{\prime \prime}+\left(1-a_{1}\right) r^{2}\left(a^{\prime}\right)^{2}+\left(6-8 a_{1}\right) r a^{\prime} \\
& \left.\left.-4\left(2 a_{1}-1\right)\right] f_{\mathcal{T}}+2 f r^{2} e^{b}\right\},
\end{aligned}
$$

where ' denotes a derivation in relation to radial coordinate $r$. Taking the metric (52) to the equations of $f(\bar{R})$ gravity in (47), we obtain

$$
\begin{aligned}
\kappa^{2} \Theta_{0}^{0}= & -\frac{e^{-b}}{4 r}\left\{4 r \frac{\mathrm{d}^{2}}{\mathrm{~d} r^{2}} f_{\bar{R}}+\left(8-2 r b^{\prime}\right) \frac{\mathrm{d}}{\mathrm{d} r} f_{\bar{R}}\right. \\
& \left.+\left[r\left(a^{\prime} b^{\prime}-2 a^{\prime \prime}-\left(a^{\prime}\right)^{2}\right)-4 a^{\prime}\right] f_{\bar{R}}+2 f r e^{b}\right\},
\end{aligned}
$$

$$
\begin{aligned}
\kappa^{2} \Theta_{1}^{1}= & \frac{e^{-b}}{4 r}\left\{\left(2 r a^{\prime}+8\right) \frac{\mathrm{d}}{\mathrm{d} r} f_{\bar{R}}\right. \\
& \left.+\left[\left(r a^{\prime}+4\right) b^{\prime}-2 r a^{\prime \prime}-r\left(a^{\prime}\right)^{2}\right] f_{\bar{R}}+2 f r e^{b}\right\},
\end{aligned}
$$

$$
\begin{aligned}
\kappa^{2} \Theta_{2}^{2}= & \kappa^{2} \Theta_{3}^{3}=\frac{e^{-b}}{2 r^{2}}\left\{2 r^{2} \frac{\mathrm{d}^{2}}{\mathrm{~d} r^{2}} f_{\bar{R}}-r\left(r b^{\prime}-r a^{\prime}-2\right) \frac{\mathrm{d}}{\mathrm{d} r} f_{\bar{R}}\right. \\
& \left.+\left(r b^{\prime}+2 e^{b}-r a^{\prime}-2\right) f_{\bar{R}}+f r^{2} e^{b}\right\} .
\end{aligned}
$$

Here first we notice that if $a_{1} \neq 1$, there exists an equation, Eq. (55), outside the diagonal for GTT, resulting in the restriction of the functional form $f(\mathcal{T})=c_{1} \mathcal{T}+c_{0}$, where $c_{0}, c_{1} \in \Re$. Then we have the same constraint to $f(T)$ gravity in this case [67].

We also see that in the limit $a_{1} \rightarrow 1,\{\mathcal{T} \rightarrow \bar{R}, f(\mathcal{T}) \rightarrow$ $\left.f(\bar{R}), f_{\mathcal{T}} \rightarrow f_{\bar{R}}\right\}$, all equations (53)-(56) for GTT are identical to $f(\bar{R})$ given in (57)-(59).
Now choose a set of non-diagonal tetrads,

$$
\left\{e_{\mu}^{a}\right\}=\left[\begin{array}{cccc}
e^{a / 2} & 0 & 0 & 0 \\
0 & e^{b / 2} \sin \theta \cos \phi & r \cos \theta \cos \phi & -r \sin \theta \sin \phi \\
0 & e^{b / 2} \sin \theta \sin \phi & r \cos \theta \sin \phi & r \sin \theta \cos \phi \\
0 & e^{b / 2} \cos \theta & -r \sin \theta & 0
\end{array}\right],
$$

and the equations to GTT in (34) provide us with

$$
\begin{aligned}
\kappa^{2} \Theta_{0}^{0}= & -\frac{e^{-b}}{4 r^{2}}\left\{4 a_{1} r^{2} \frac{\mathrm{d}^{2}}{\mathrm{~d} r^{2}} f_{\mathcal{T}}-\left(2 a_{1} r^{2} b^{\prime}-8\left(a_{1}-1\right) r e^{b / 2}\right.\right. \\
& -8 r) \frac{\mathrm{d}}{\mathrm{d} r} f_{\mathcal{T}}+\left[\left(a_{1} r^{2} a^{\prime}+4\left(a_{1}-1\right) r\right) b^{\prime}\right. \\
& +\left(4\left(a_{1}-1\right) r a^{\prime}+8\left(a_{1}-1\right)\right) e^{b / 2}-a_{1} r^{2}\left(2 a^{\prime \prime}+\left(a^{\prime}\right)^{2}\right) \\
& \left.\left.-\left(8 a_{1}-4\right) r a^{\prime}-8\left(a_{1}-1\right)\right] f_{\mathcal{T}}+2 f r^{2} e^{b}\right\}, \\
\kappa^{2} \Theta_{1}^{1}= & \frac{e^{-3 b / 2}}{4 r^{2}}\left\{2 a_{1} r\left(r a^{\prime}+4\right) e^{b / 2} \frac{\mathrm{d}}{\mathrm{d} r} f_{\mathcal{T}}\right. \\
+ & {\left[a_{1} r\left(a^{\prime} r+4\right) e^{b / 2} b^{\prime}+\left[4\left(a_{1}-1\right) r a^{\prime}+8\left(a_{1}-1\right)\right] e^{b}\right.} \\
& -\left(a_{1} r^{2}\left(2 a^{\prime \prime}+\left(a^{\prime}\right)^{2}\right)-8\left(1-a_{1}\right) r a^{\prime}\right. \\
& \left.\left.\left.-8\left(1-a_{1}\right)\right) e^{b / 2}\right] f_{\mathcal{T}}+2 f r^{2} e^{3 b / 2}\right\}, \\
\kappa^{2} \Theta_{2}^{2}= & \kappa^{2} \Theta_{3}^{3}=\frac{e^{-b}}{4 r^{2}}\left\{4 a_{1} r^{2} \frac{\mathrm{d}^{2}}{\mathrm{~d} r^{2}} f_{\mathcal{T}}\right. \\
& -\left(2 a_{1} b^{\prime}-4\left(a_{1}-1\right) r e^{b / 2}-2 r^{2} a^{\prime}-4 r\right) \frac{\mathrm{d}}{\mathrm{d} r} f_{\mathcal{T}} \\
& +\left[\left(\left(a_{1}-1\right) r^{2} a^{\prime}+\left(4 a_{1}-2\right) r\right) b^{\prime}+4 e^{b}\right. \\
& +\left(4\left(a_{1}-1\right) r a^{\prime}+8\left(a_{1}-1\right)\right) e^{b / 2} \\
& -2\left(a_{1}-1\right) r^{2} a^{\prime \prime}-\left(a_{1}-1\right) r^{2}\left(a^{\prime}\right)^{2}+\left(6-8 a_{1}\right) r a^{\prime} \\
& \left.\left.-\left(8 a_{1}-4\right)\right] f_{\mathcal{T}}+2 f r^{2} e^{b}\right\} . \\
& \left.-b^{\prime}\right)
\end{aligned}
$$

We can then see that in this case the equations of motion are diagonal. But equivalence of GTT with $f(\bar{R})$ gravity is only given in the limit $a_{1} \rightarrow 1$, when Eqs. (57)-(59) and (61)-(63) are identical.

\section{Equivalence between GTT and a particular case of the $f(T, B)$ gravity}

In this section we make an important observation. When we were finishing the calculation of the non-diagonal tetrads case of the previous subsection, we noted that a collaboration have submitted exactly the same idea as our work here. The so-call $f(T, B)$ gravity [68], with $B=-2 \bar{\nabla}^{\mu} T_{\mu \nu}^{v}$, is a more general theory than presented here, where the algebraic function is 
contained in the action; it may be any analytic function of the variables $T$ and $B$. We noted then that the equivalence of this theory with the $f(\bar{R})$ gravity is given only for the specific functional form $f(T, B) \equiv f(-T+B)=f(\bar{R})$. Compared to our theory, we see that GTT is a particular case of $f(T, B)$ gravity, when $f(T, B) \equiv f\left(-T+a_{1} B\right)=f(\mathcal{T})$. We can show this again explicitly using the equations of motion.

The equation of motion for $f(T, B)$ gravity is given by

$$
\begin{aligned}
& 2 \delta_{v}^{\lambda} \bar{\square} f_{B}-2 \bar{\nabla}^{\lambda} \bar{\nabla}_{\nu} f_{B}+B f_{B} \delta_{v}^{\lambda} \\
& \quad+4 \partial_{\mu}\left(f_{B}+f_{T}\right) S_{v}^{\mu \lambda}+4 e^{-1} e_{\nu}^{a} \partial_{\mu}\left(e e_{a}^{\beta} S_{\beta}^{\mu \lambda}\right) f_{T} \\
& \quad-4 f_{T} T_{\mu \nu}^{\sigma} S_{\sigma}^{\lambda \mu}-f \delta_{v}^{\lambda}=2 \kappa^{2} \Theta_{\nu}^{\lambda} .
\end{aligned}
$$

The first observation here is that this theory does not fall under $f(T)$ gravity in general, as well as our GTT, as mentioned at the end of Sect. 3. Taking $f(T, B) \equiv f(T)$, thus $f_{B}=0$, the equation of motion (64), using the identity (16), becomes

$4\left(\partial_{\mu} f_{T}\right) S_{v}^{\mu \lambda}-2 f_{T} G_{v}^{\lambda}+\delta_{v}^{\lambda}\left(T f_{T}-f\right)=2 \kappa^{2} \Theta_{v}^{\lambda}$.

This equation is not equal to (17) for $f(T)$ gravity, and cannot fall under GR when $f(T) \equiv T-2 \Lambda$, due to the sign. This shows that the $f(T, B)$ gravity also does not return to $f(T)$ gravity in general.

Now we can show that in the particular case $f\left(-T+a_{1} B\right)$ this theory falls under our GTT. We take the FLRW metric (44) with diagonal tetrads $\left[e_{\mu}^{a}\right]=\operatorname{diag}[1, a, a, a]$, and the equations of motion (64) provide us with

$$
\begin{aligned}
\kappa^{2} \Theta_{0}^{0}= & -\frac{1}{2 a^{2}}\left\{12(\dot{a})^{2} f_{T}-6 a \dot{a} \frac{\mathrm{d}}{\mathrm{d} t} f_{B}\right. \\
& \left.+2\left[3 a \ddot{a}+6(\dot{a})^{2}\right] f_{B}+f a^{2}\right\}, \\
\kappa^{2} \Theta_{1}^{1}= & \kappa^{2} \Theta_{2}^{2}=\kappa^{2} \Theta_{3}^{3}=\frac{1}{2 a^{2}}\left\{4 a(\dot{a})\left(\frac{\mathrm{d}}{\mathrm{d} t} f_{T}\right)\right. \\
& +\left[4 a \ddot{a}+8(\dot{a})^{2}\right] f_{T}-2 a^{2}\left(\frac{\mathrm{d}^{2}}{\mathrm{~d} t^{2}} f_{B}\right) \\
& \left.+\left[6 a \ddot{a}+12(\dot{a})^{2}\right] f_{B}+f a^{2}\right\} .
\end{aligned}
$$

Now identifying $f\left(-T+a_{1} B\right)=f(\mathcal{T})$, recalling that $\mathcal{T}$ is given in (19),

$$
\begin{aligned}
& f_{T}=\frac{\partial f}{\partial T}=\frac{\partial \mathcal{T}}{\partial T} \frac{\mathrm{d} f}{\mathrm{~d} \mathcal{T}}=-\frac{\mathrm{d} f}{\mathrm{~d} \mathcal{T}}, \\
& f_{B}=\frac{\partial f}{\partial B}=\frac{\partial \mathcal{T}}{\partial B} \frac{\mathrm{d} f}{\mathrm{~d} \mathcal{T}}=a_{1} \frac{\mathrm{d} f}{\mathrm{~d} \mathcal{T}} .
\end{aligned}
$$

We see that Eqs. (66) and (67) are identical with GTT (45) and (46), thus showing the equivalence between the theories.

We can also confirm this by choosing the spherical symmetry for the metric (52), first for diagonal tetrads $\left[e_{\mu}^{a}\right]=$ $\operatorname{diag}\left[e^{a / 2}, e^{b / 2}, r, r \sin \theta\right]$, thus, Eq. (64) provide us with

$$
\begin{aligned}
\kappa^{2} \Theta_{0}^{0}= & \frac{e^{-b}}{4 r^{2}}\left\{8 r \frac{\mathrm{d}}{\mathrm{d} r} f_{T}-2\left[2 r b^{\prime}+2 e^{b}-2 r a^{\prime}-4\right] f_{T}\right. \\
& -2 r^{2}\left[2 \frac{\mathrm{d}}{\mathrm{d} r}-b^{\prime}\right] \frac{\mathrm{d}}{\mathrm{d} r} f_{B} \\
& +\left[-r\left(r a^{\prime}+4\right) b^{\prime}-4 e^{b}+r\left(2 r a^{\prime \prime}+r\left(a^{\prime}\right)^{2}+8 a^{\prime}\right)\right. \\
& \left.+8] f_{B}-2 f r^{2} e^{b}\right\}, \\
\kappa^{2} \Theta_{1}^{1}= & \frac{e^{-b}}{4 r^{2}}\left\{2\left[2 e^{b}-4 r a^{\prime}-4\right] f_{T}+2 r\left[r a^{\prime}+4\right] \frac{\mathrm{d}}{\mathrm{d} r} f_{B}\right. \\
& +\left[r\left(r a^{\prime}+4\right) b^{\prime}+4 e^{b}-r\left(2 r a^{\prime \prime}-r\left(a^{\prime}\right)^{2}-8 a^{\prime}\right)\right. \\
& \left.-8] f_{B}+2 r^{2} f e^{b}\right\}, \\
\kappa^{2} \Theta_{2}^{1}= & -\frac{1}{r^{2} \sin \theta}\left[\cos \theta \frac{\mathrm{d}}{\mathrm{d} r} f_{T}+\cos \theta \frac{\mathrm{d}}{\mathrm{d} r} f_{B}\right]=0, \\
\kappa^{2} \Theta_{2}^{2}= & \kappa^{2} \Theta_{3}^{3}=\frac{e^{-b}}{4 r^{2}}\left\{2 r\left(r a^{\prime}+2\right) \frac{\mathrm{d}}{\mathrm{d} r} f_{T}\right. \\
& +\left[-r\left(r a^{\prime}+2\right) b^{\prime}+r^{2}\left(2 a^{\prime \prime}+\left(a^{\prime}\right)^{2}\right)+6 r a^{\prime}+4\right] f_{T} \\
& -2 r^{2}\left[2 \frac{\mathrm{d}}{\mathrm{d} r}-b^{\prime}\right] \frac{\mathrm{d}}{\mathrm{d} r} f_{B}+\left[-r\left(r a^{\prime}-4\right) b^{\prime}\right. \\
& \left.\left.-4 e^{b}+r^{2}\left(2 a^{\prime \prime}+\left(a^{\prime}\right)^{2}\right)+8 r a^{\prime}+8\right] f_{B}-2 f r^{2} e^{b}\right\} .
\end{aligned}
$$

Again we have the equivalence of the equations of motion (69)-(72) with (53)-(56), for the identifications $f(T, B)=$ $f(\mathcal{T})$ and $(68)$.

By taking the choice of non-diagonal tetrads (60), the equations of motion from $f(T, B)$ gravity (64) provide us with

$$
\begin{aligned}
\kappa^{2} \Theta_{0}^{0}= & -\frac{e^{-5 b / 2}}{4 r^{2}}\left\{8 r\left[e^{2 b}-e^{3 b / 2}\right] \frac{\mathrm{d}}{\mathrm{d} r} f_{T}\right. \\
& +\left[4 r e^{3 b / 2} b^{\prime}+2\left(2 r a^{\prime}+4\right) e^{2 b}-4\left(r a^{\prime}+2\right) e^{3 b / 2}\right] f_{T} \\
& +\left[4 r^{2} e^{3 b / 2} \frac{\mathrm{d}}{\mathrm{d} r}+\left(8 r e^{2 b}-2 r^{2} e^{3 b / 2}\right)\right] \frac{\mathrm{d}}{\mathrm{d} r} f_{B} \\
& +\left[r\left(r a^{\prime}+4\right) e^{3 b / 2} b^{\prime}+4\left(r a^{\prime}+2\right) e^{2 b}\right. \\
& \left.-\left(2 r^{2} a^{\prime \prime}+r\left(a^{\prime}\right)^{2}+8 r a^{\prime}+8\right) e^{3 b / 2}\right] f_{B} \\
& \left.+2 f r^{2} e^{5 b / 2}\right\}, \\
\kappa^{2} \Theta_{1}^{1}= & \frac{e^{-5 b / 2}}{4 r^{2}}\left\{\left[4\left(r a^{\prime}+2\right) e^{2 b}-8\left(r a^{\prime}+1\right) e^{3 b / 2}\right] f_{T}\right. \\
& +2 r\left(r a^{\prime}+4\right) e^{3 b / 2} \frac{\mathrm{d}}{\mathrm{d} r} f_{B} \\
& +\left[r\left(r a^{\prime}+4\right) e^{3 b / 2} b^{\prime}+4\left(r a^{\prime}+2\right) e^{2 b}-\left(2 r^{2} a^{\prime \prime}\right.\right. \\
& \left.\left.\left.+r^{2}\left(a^{\prime}\right)^{2}+8 r a^{\prime}+8\right) e^{3 b / 2}\right] f_{B}+2 f r^{2} e^{5 b / 2}\right\}
\end{aligned}
$$




$$
\begin{aligned}
\kappa^{2} \Theta_{2}^{2}= & \kappa^{2} \Theta_{3}^{3}=\frac{e^{-5 b / 2}}{4 r^{2}}\left\{\left[4 r e^{2 b}-2 r\left(r a^{\prime}+2\right) e^{3 b / 2}\right] \frac{\mathrm{d}}{\mathrm{d} r} f_{T}\right. \\
& +\left[r\left(r a^{\prime}+2\right) e^{3 b / 2} b^{\prime}-4 e^{5 b / 2}+4\left(r a^{\prime}+2\right) e^{2 b}\right. \\
& \left.-\left(2 r^{2} a^{\prime \prime}+r^{2}\left(a^{\prime}\right)^{2}+6 r a^{\prime}+4\right) e^{3 b / 2}\right] f_{T} \\
& +\left[4 r^{2} e^{3 b / 2} \frac{\mathrm{d}}{\mathrm{d} r}+4 r e^{2 b}-2 r^{2} e^{3 b / 2} b^{\prime}\right] \frac{\mathrm{d}}{\mathrm{d} r} f_{B} \\
& +\left[r\left(r a^{\prime}+4\right) e^{3 b / 2} b^{\prime}+4\left(r a^{\prime}+2\right) e^{2 b}\right. \\
& \left.-\left(2 r^{2} a^{\prime \prime}+r^{2}\left(a^{\prime}\right)^{2}+8 r a^{\prime}+8\right) e^{3 b / 2}\right] f_{B} \\
& \left.+2 f r^{2} e^{5 b / 2}\right\} .
\end{aligned}
$$

Just as before, making the identifications $f(T, B)=f(\mathcal{T})$ and (68), Eqs. (73)-(75) are identical to GTT in (61)-(63), confirming again the equivalence of these theories.

\section{Applications to GTT}

\subsection{Reconstruction for de Sitter Universe}

A method to obtain the functional form of the algebraic function $f(\mathcal{T})$ is the so-called reconstruction. This method consists of specifying a model that fixes the matter content of the theory in terms of the scalar $\mathcal{T}$, allowing one to reconstruct the functional form of $f(\mathcal{T})$ through the equations of motion of the theory.

We will choose the particular case of a flat FLRW metric in which $a(t)=a_{0} \exp \left[H_{0}\left(t-t_{0}\right)\right], a_{0}, H_{0}, t_{0} \in \Re_{+}$; it provides us with the model of de Sitter universe, where $H(t)=\dot{a} / a=H_{0}$. In this case, using (19), we have $H_{0}(\mathcal{T})=\sqrt{\mathcal{T} /\left[6\left(1-3 a_{1}\right)\right]}, \dot{H} \equiv 0$ and $(\mathrm{d} / \mathrm{d} t) f_{\mathcal{T}}=$ $f_{\mathcal{T} \mathcal{T}}(\mathrm{d} / \mathrm{d} t) \mathcal{T} \equiv 0$. Knowing that $\kappa^{2} \Theta_{0}^{0}=\kappa^{2} \rho=3 H_{0}^{2}$, Eq. (45) provides us with

$3\left[H_{0}(\mathcal{T})\right]^{2}=3\left[H_{0}(\mathcal{T})\right]^{2}\left(2-3 a_{1}\right) f_{\mathcal{T}}(\mathcal{T})-\frac{1}{2} f(\mathcal{T})$,

and integrating with respect to that $\mathcal{T}$ results in

$$
f(\mathcal{T})=\mathcal{T}+\left[\left(2-3 a_{1}\right) \mathcal{T}\right]^{\left(1-3 a_{1}\right) /\left(2-3 a_{1}\right)} c_{1}, c_{1} \in \Re .
$$

\subsection{Spherially symmetric type-de Sitter solution}

We here take the limit $a_{1} \rightarrow 0$ in (34), which after using the identity (16) and considering $\mathcal{T} \rightarrow-T$ results in

$$
f_{T}(-T) G_{\omega}^{\sigma}+\frac{1}{2} \delta_{\omega}^{\sigma}\left[f(-T)-T f_{T}(-T)\right]=-\kappa^{2} \Theta_{\omega}^{\sigma} .
$$

As in $f(\bar{R})$ gravity [69], we can consider the very specific case where $\bar{R} \equiv \bar{R}_{0}=-T_{0}+B_{0}, \mathcal{T} \equiv \mathcal{T}_{0}=-T_{0}+a_{1} B_{0}=$ $-T_{0}$, with $\bar{R}_{0}, T_{0}, B_{0} \in \Re$ and $B_{0}$ is defined by (19). In the case of a perfect fluid $\Theta_{v}^{\lambda}=\operatorname{diag}\left[\rho_{0},-p_{0},-p_{0},-p_{0}\right]$, and $\partial_{\mu} f_{T}=f_{T T} \partial_{\mu} T_{0} \equiv 0$, which results in the equations

$$
\bar{R}_{\omega}^{\sigma}=-\frac{\kappa^{2}}{f_{T_{0}}\left(-T_{0}\right)} \Theta_{\omega}^{\sigma}+\frac{1}{2} \delta_{\omega}^{\sigma}\left[\bar{R}_{0}+T_{0}-\frac{f\left(-T_{0}\right)}{f_{T_{0}}\left(-T_{0}\right)}\right],
$$

which taking the trace results in

$$
B_{0}=\frac{\kappa^{2}}{f_{T_{0}}\left(-T_{0}\right)}\left(\rho_{0}-3 p_{0}\right)+2 \frac{f\left(-T_{0}\right)}{f_{T_{0}}\left(-T_{0}\right)}-T_{0} .
$$

Considering now the line element (52), for $b(r)=-a(r)$ and $p_{0}=-\rho_{0}$ (type dark energy), we can integrate the equations of motion (79), where we get the following solution:

$$
\begin{aligned}
a(r)= & -b(r)=\log \left[1+\frac{c_{1}}{2 f_{T_{0}}\left(-T_{0}\right) r}\right. \\
& \left.+\frac{f\left(-T_{0}\right)-f_{T_{0}}\left(-T_{0}\right) T_{0}+2 \kappa^{2} \rho_{0}}{6 f_{T_{0}}\left(-T_{0}\right)} r^{2}\right] .
\end{aligned}
$$

This is a static de Sitter type solution where we can identify the effective cosmological constant $\left(-\Lambda_{\text {eff }} / 3\right)=\left[f\left(-T_{0}\right)-\right.$ $\left.f_{T_{0}}\left(-T_{0}\right) T_{0}+2 \kappa^{2} \rho_{0}\right] /\left[6 f_{T_{0}}\left(-T_{0}\right)\right]$. A de Sitter type solution was also previously obtained in $f(\bar{R})$ gravity for $a(r)=$ $-b(r)$ and $\bar{R}=\bar{R}_{0}$ [69]. We emphasize here that this solution boils down to a different theory from $f(T)$ gravity, because GTT does not fall under $f(T)$ gravity for $a_{1} \rightarrow 0$, except for the special case where $f(T)$ is an odd analytic function, that is, $f(-T)=-f(T)$.

\subsection{Evolution for the state parameter of the dark energy}

A good test for our theory is the evolution of a model of the universe. This can discard or keep a theory depending on whether it is in agreement with the observational data.

Let us follow the procedure found in [70] to determine the state parameter $\omega_{\mathrm{DE}}$. For a universe permeated by a perfect fluid, of which the equation of state is governed by $p=\omega \rho$, we can rewrite the equations of motion (45) and (46) as

$$
\begin{aligned}
& 3 H^{2}=\kappa^{2} G_{\text {eff }}\left(\rho_{m}+\rho_{\mathrm{DE}}\right), \\
& H=\frac{\dot{a}}{a}, \\
& G_{\mathrm{eff}}=\frac{1}{\left(3 a_{1}-2\right) f_{\mathcal{T}}}, \\
& \rho_{\mathrm{DE}}=\frac{1}{\kappa^{2}}\left[3 a_{1}\left(\dot{H} f_{\mathcal{T}}-H \dot{f}_{\mathcal{T}}\right)-\frac{1}{2} f\right], \\
& \dot{H}=-\frac{\kappa^{2}}{2} G_{\mathrm{eff}}\left(p_{m}+p_{\mathrm{DE}}+\rho_{m}+\rho_{\mathrm{DE}}\right), \\
& p_{\mathrm{DE}}=\frac{1}{\kappa^{2}}\left[a_{1} \ddot{f}_{\mathcal{T}}+H \dot{f}_{\mathcal{T}}-\frac{1}{2} f\right] .
\end{aligned}
$$




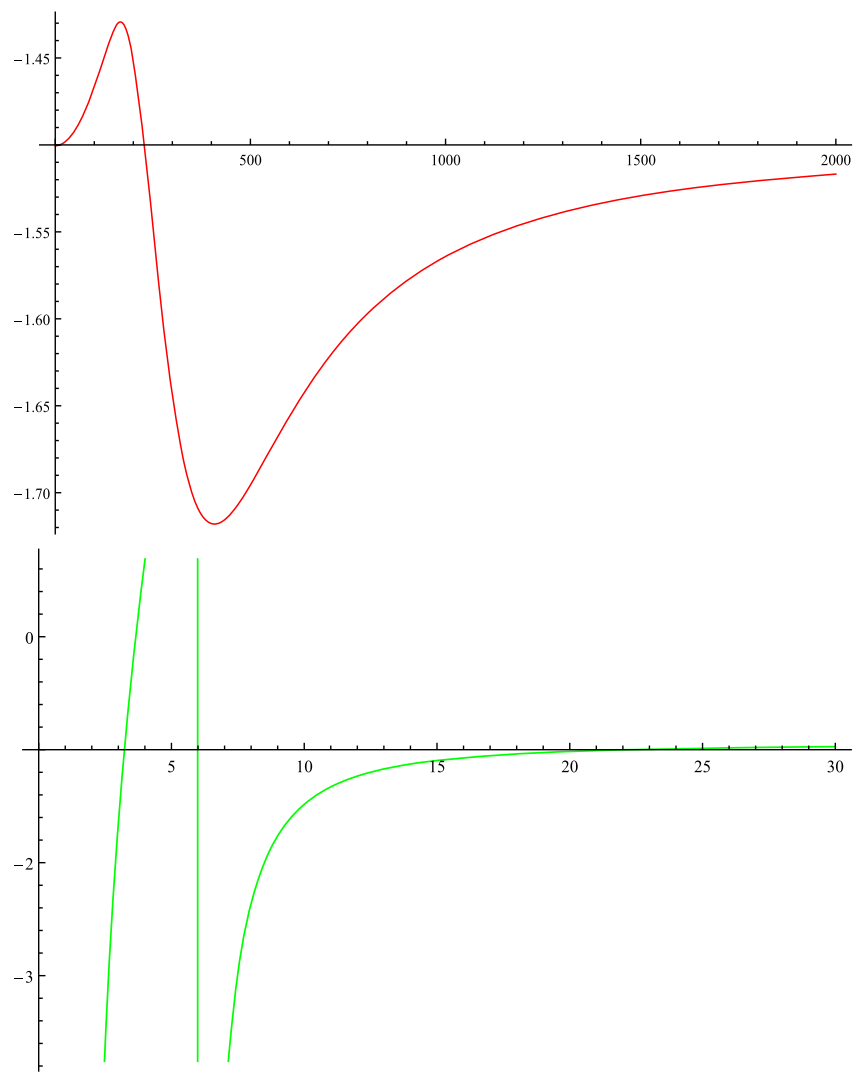

Fig. 1 Representation of the temporal evolution of $\omega_{\mathrm{DE}}(t)$

Now we can defining the state parameter of dark energy by

$\omega_{\mathrm{DE}}=\frac{p_{\mathrm{DE}}}{\rho_{\mathrm{DE}}}=\frac{a_{1} \ddot{f}_{\mathcal{T}}+H \dot{f}_{\mathcal{T}}-\frac{1}{2} f}{3 a_{1}\left(\dot{H} f_{\mathcal{T}}-H \dot{f}_{\mathcal{T}}\right)-\frac{1}{2} f}$.

We now assume an exponential model [70] defined by

$f(\mathcal{T})=\mathcal{T}-\beta \mathcal{T}_{s}\left(1-\exp \left[\frac{\mathcal{T}}{\mathcal{T}_{s}}\right]\right)$.

We will now test for a solution of power law type,

$a(t)=t^{\alpha}, H(t)=\frac{\alpha}{t}$.

We can show that (86) is a solution of the equations of motion (82) and (83) if the matter part is given by the expressions

$$
\begin{aligned}
\rho_{m t}= & \frac{1}{2 t^{4} \mathcal{T}_{s} \kappa^{2}}\left\{e ^ { \frac { 6 \alpha [ a _ { 1 } + \alpha ( 1 - 2 a _ { 1 } ) ] } { \mathcal { T } _ { s } t ^ { 2 } } } \left[\mathcal{T}_{s}^{2} t^{4}+6 \alpha \mathcal{T}_{s} t^{2}\left(a_{1}\right.\right.\right. \\
& \left.\left.+\alpha\left(3 a_{1}-2\right)\right)+72 a_{1} \alpha^{2}\left(a_{1}(2 \alpha-1)-\alpha\right)\right] \beta \\
& \left.+\mathcal{T}_{s} t^{2}\left[-6 \alpha^{2}+6 \alpha a_{1}(2+\alpha)-\beta \mathcal{T}_{s} t^{2}\right]\right\}, \\
p_{m t}= & \frac{1}{2 \mathcal{T}_{s}^{2} \kappa^{2} t^{6}}\left\{e ^ { \frac { 6 \alpha [ a _ { 1 } + \alpha ( 1 - 2 a _ { 1 } ) ] } { \mathcal { T } _ { s } t ^ { 2 } } } \left[-\mathcal{T}_{s}^{3} t^{6}\right.\right. \\
& +2 \mathcal{T}_{s}^{2} \alpha t^{4}\left(-4+3 a_{1}(2-3 \alpha)+6 \alpha\right) \\
& +288 a_{1} \alpha^{2}\left(a_{1}+\alpha\left(1-2 a_{1}\right)\right)^{2}
\end{aligned}
$$

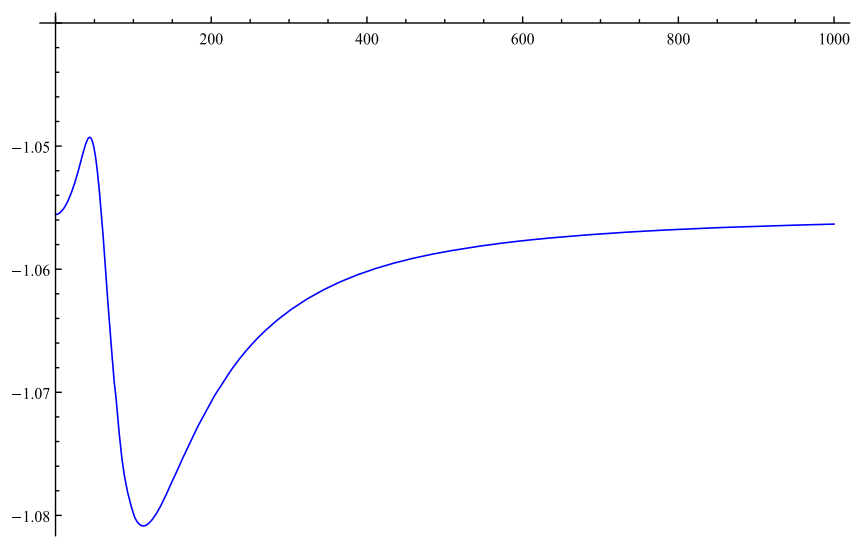

$$
\begin{aligned}
& \left.-24 \mathcal{T}_{s} t^{2} \alpha\left(3 a_{1}-\alpha\right)\left(-\alpha+a_{1}(2 \alpha-1)\right)\right] \beta \\
& \left.+\mathcal{T}_{s}^{2} t^{4}\left[2 \alpha\left(-4-3 a_{1}(\alpha-1)+3 \alpha\right)+\beta \mathcal{T}_{s} t^{2}\right]\right\} .
\end{aligned}
$$

Figure 1 is the temporal evolution of the state parameter $\omega_{\mathrm{DE}}$ of the dark energy. The red curve is obtained with a constant given by $\left\{\alpha=2, \beta=1, \mathcal{T}_{s}=H_{0} \Omega_{m}^{(0)} / \beta, H_{0}=\right.$ $\left.0.75, \Omega_{m}^{(0)}=0.23, a_{1}=1000\right\}$, where we can see that the fluid is always phantom $\omega_{\mathrm{DE}}<-1$. The blue curve is obtained with a constant given by $\left\{\alpha=20, \beta=1, \mathcal{T}_{s}=\right.$ $\left.H_{0} \Omega_{m}^{(0)} / \beta, H_{0}=0.75, \Omega_{m}^{(0)}=0.23, a_{1}=1\right\}$, where we see that the fluid is always phantom $\omega_{\mathrm{DE}}<-1$, but it fluctuates approximately between the values -1.05 and -1.08 . The most interesting case is the green curve obtained for the constants $\left\{\alpha=2, \beta=1, \mathcal{T}_{s}=H_{0} \Omega_{m}^{(0)} / \beta, H_{0}=\right.$ $\left.0.75, \Omega_{m}^{(0)}=0.23, a_{1}=0.1\right\}$. In this case we see that the fluid begins in a rather phantom phase, going through another quintessence type phase, heading toward behavior of baryonic matter $(\omega>0)$, and finally returning to the phantom phase. The result is that the current accelerated expansion of the universe and the crossing of the phantom divide from the phantom phase to the non-phantom (quintessence) one can be realized as well as in [70]. 


\subsection{Thermodynamics for an apparent horizon}

A further application is for thermodynamics of the apparent horizon in cosmology with the FLRW metric. We can follow the formulation given in [71].

We can establish a similar equation of continuity, deriving over time (82) and using (83)

$$
\begin{aligned}
\dot{\rho}_{m} & +\dot{\rho}_{\mathrm{DE}}+3 H\left(\rho_{m}+\rho_{\mathrm{DE}}+p_{m}+p_{\mathrm{DE}}\right) \\
= & 3 H^{2} \frac{\mathrm{d}}{\mathrm{d} t}\left(\frac{1}{G_{\text {eff }}}\right) .
\end{aligned}
$$

Whereas the baryonic matter is conserved $\left(\dot{\rho}_{m}+3 H\left(\rho_{m}+\right.\right.$ $\left.p_{m}\right) \equiv 0$ ), we see that dark energy is not conserved, yielding the interpretation that it is a system out of equilibrium with entropy production (non-equilibrium thermodynamics). Following exactly the same steps as in [71], we can establish the first law of thermodynamics,

$$
\begin{aligned}
T_{A} \mathrm{~d} S_{A}+T_{A} \mathrm{~d} S_{p} & =-\mathrm{d} E_{M S}+W \mathrm{~d} V, \\
T_{A} \mathrm{~d} S_{p} & =-\frac{1}{2} \hat{r}_{A}\left(1+2 \pi \hat{r}_{A} T_{A}\right) \mathrm{d}\left(\frac{1}{G_{\text {eff }}}\right),
\end{aligned}
$$

where $T_{A}$ the temperature of the apparent horizon, $\mathrm{d} S_{A}$ is the entropy of the apparent horizon, $\mathrm{d} S_{p}$ is the produced entropy, $\mathrm{d} E_{M S}$ is the Misner-Sharp energy, $W$ the work, and $d V$ the volume element of the apparent horizon. Here it is clearly seen that the first law of thermodynamics is consistent for entropy production associated with an effective Newton constant $G_{\text {eff }}$, given in (82), for which for the linear case of $f(\mathcal{T})$ the entropy production vanishes and the system goes back to equilibrium.

If we take the same model as the previous section, i.e. (85) and (86), we can explicitly show the time dependence of the effective Newton constant in (82),

$G_{\text {eff }}=\left(3 a_{1}-2\right)^{-1}\left\{1+\beta e^{\frac{6 \alpha\left[a_{1}+\alpha\left(1-2 a_{1}\right)\right]}{\mathcal{T}_{s} t^{2}}}\right\}^{-1}$.

Here two important observations are in order. The first is that it gets an explicit dependence of the first law of thermodynamics corresponding to the specific choice of the value on $a_{1}$ in (91). The second is that by taking the particular value $\beta \equiv 0$ in (91), clearly we have $G_{\text {eff }}=\left(3 a_{1}-2\right)^{-1}$, which again shows the dependence of the theory in relation to the specific value of $a_{1}$, and from (91), (85) and (90) we return to the linear theory, where there is no entropy production.

\section{Conclusion}

We construct a theory that describes the gravitational interaction through effects of torsion of space-time. This theory generalizes the teleparallel theory, keeping the invariance by both local Lorentz transformations as general coordinates transformations for a particular case.

The action of our theory is described by a general algebraic function that depends on a tensorial scalar $\mathcal{T}$, which is classified by a real parameter $a_{1}$. Our theory falls exactly under $f(\bar{R})$ gravity when we take the limit $a_{1} \rightarrow 1$. This is shown from the equations of motion of the two theories.

We show explicitly through the equations of motion of our theory that it is also equivalent to the recently found $f(T, B)$ gravity, when $f(T, B)=f\left(-T+a_{1} B\right)$.

We make two small applications of our theory, reconstructing the action for the particular case of a de Sitter universe for the flat FLRW metric, with a set of diagonal tetrads, and for obtaining a static de Sitter type solution. We also analyze the evolution of the state parameter of the dark energy and the first law of thermodynamics for the apparent horizon.

Our theory is a good scenario for an attempt to explain the accelerated expansion of our universe, by modifying the usual teleparallel gravitation, or by analogy to Einstein gravity. The real parameter $a_{1}$ which classifies which theory GTT describes is crucial to any consideration of cosmological phenomena. We also expect new solutions of black holes to arise through our theory, in which one may also suggest to shed some light on the so-called dark matter explanation of local effects of gravitation.

Another perspective is to show the stability of the three solutions discussed here. This should be a topic for future work.

Acknowledgments Manuel E. Rodrigues thanks UFPA, Edital 04/2014 PROPESP, and CNPq, Edital MCTI/CNPQ/Universal 14/2014, for partial financial support. The authors thank Prof. José W. Maluf for their cooperation during the preparation of this work.

Open Access This article is distributed under the terms of the Creative Commons Attribution 4.0 International License (http://creativecomm ons.org/licenses/by/4.0/), which permits unrestricted use, distribution, and reproduction in any medium, provided you give appropriate credit to the original author(s) and the source, provide a link to the Creative Commons license, and indicate if changes were made.

Funded by SCOAP . $^{3}$

\section{References}

1. A. Riess et al., Observational evidence from supernovae for an accelerating universe and a cosmological constant. Astron. J. 116, 1009 (1998). arXiv:astro-ph/9805201

2. S. Perlmutter et al., Measurements of and from 42 HighRedshift Supernovae. Astrophys. J. 517, 565 (1999). arXiv:astro-ph/9812133

3. J. Tonry et al., Cosmological results from high-z supernovae. Astrophys. J. 594, 1 (2003). arXiv: astro-ph/0305008

4. J. Plebanski, A. Krasinski, An introduction to general relativity and cosmology (Cambridge University Press, New York, 2006)

5. S. Nojiri, S.D. Odintsov, Introduction to modified gravity and gravitational alternative for dark energy. ECONF C0602061, 06 (2006) 
6. S. Nojiri, S.D. Odintsov, Int. J. Geom. Meth. Mod. Phys. 4, 115146 (2007). arXiv:hep-th/0601213

7. T.P. Sotiriou, V. Faraoni, $\mathrm{f}(\mathrm{R})$ Theories Of Gravity. Rev. Mod. Phys. 82, 451-497 (2010). arXiv:0805.1726

8. A. De Felice, S. Tsujikawa, $\mathrm{f}(\mathrm{R})$ theories. Living Rev. Rel. 13, 3 (2010). arXiv: 1002.4928

9. T. Clifton, P.G. Ferreira, A. Padilla, C. Skordis, Modified gravity and cosmology. Phys. Rep. 513(1), 1-189 (2012). arXiv:1106.2476

10. T. Harko, F.S.N. Lobo, S. Nojiri, S.D. Odintsov, f(R, T) gravity. Phys. Rev. D 84, 024020 (2011). arXiv:1104.2669

11. M. Jamil, D. Momeni, M. Raza, R. Myrzakulov, Reconstruction of some cosmological models in $\mathrm{f}(\mathrm{R}, \mathrm{T})$ gravity. Eur. Phys. J. C 72, 1999 (2012). arXiv:1107.5807

12. F.G. Alvarenga, A. de la Cruz-Dombriz, M.J.S. Houndjo, M.E. Rodrigues, D. Sez-Gmez, Reconstruction of some cosmological models in $\mathrm{f}(\mathrm{R}, \mathrm{T})$ gravity, Phys.Rev. D 87(10), 103526 (2013)

13. F.G. Alvarenga, A. de la Cruz-Dombriz, M.J.S. Houndjo, M.E. Rodrigues, D. Sez-Gmez, Reconstruction of some cosmological models in f(R,T) gravity, Phys.Rev. D 87(12), 129905 (2013). arXiv:1302.1866

14. K. Bamba, C.-Q. Geng, S. Nojiri, S.D. Odintsov, Equivalence of modified gravity equation to the Clausius relation. Europhys. Lett. 89, 50003 (2010). arXiv:0909.4397

15. M.J.S. Houndjo, M.E. Rodrigues, D. Momeni, R. Myrzakulov, Exploring cylindrical solutions in modified $\mathrm{f}(\mathrm{G})$ gravity. Can. J. Phys. 92(12), 1528-1540 (2014). arXiv:1301.4642

16. M.E. Rodrigues, M.J.S. Houndjo, D. Momeni, R. Myrzakulov, A Type of Levi-Civita's solution in modified Gauss-Bonnet gravity. Can. J. Phys. 92(2), 173-176 (2014). arXiv: 1212.4488

17. K. Bamba, S.D. Odintsov, L. Sebastiani, S. Zerbini, Finite-time future singularities in modified Gauss-Bonnet and $\mathrm{F}(\mathrm{R}, \mathrm{G})$ gravity and singularity avoidance. Eur. Phys. J. C 67, 295-310 (2010). arXiv:0911.4390

18. S. Nojiri, S.D. Odintsov, A. Toporensky, P. Tretyakov, Reconstruction and deceleration-acceleration transitions in modified gravity. Gen. Relativ. Gravit. 42, 1997-2008 (2010). arXiv:0912.2488

19. G. Cognola, E. Elizalde, S. Nojiri, S.D. Odintsov, S. Zerbini, Dark energy in modified Gauss-Bonnet gravity: late-time acceleration and the hierarchy problem. Phys. Rev. D 73, 084007 (2006). arXiv:hep-th/0601008

20. E. Elizalde, R. Myrzakulov, V.V. Obukhov, D. Sez-Gmez, LambdaCDM epoch reconstruction from $\mathrm{F}(\mathrm{R}, \mathrm{G})$ and modified GaussBonnet gravities. Class. Quant. Gravit. 27, 095007 (2010). arXiv: 1001.3636

21. A. De Felice, T. Suyama, Vacuum structure for scalar cosmological perturbations in Modified Gravity Models. JCAP 0906, 034 (2009). arXiv:0904.2092

22. A. De Felice, S. Tsujikawa, Construction of cosmologically viable $f(G)$ gravity models. Phys. Lett. B 675, 1-8 (2009). arXiv:0810.5712

23. S. Nojiri, S.D. Odintsov, Modified Gauss-Bonnet theory as gravitational alternative for dark energy. Phys. Lett. B 631, 1-6 (2005). arXiv:hep-th/0508049

24. S. Nojiri, S.D. Odintsov, Modified gravity with negative and positive powers of the curvature: unification of the inflation and of the cosmic acceleration. Phys. Rev. D 68, 123512 (2003). arXiv:hep-th/0307288

25. S.D. Odintsov, D. Saez-Gomez, $f\left(R, \Theta, R_{\mu \nu} \Theta^{\mu \nu}\right)$ gravity phenomenology and $\Lambda$ CDM universe, Phys. Lett. B 725, 437-444 (2013). arXiv:1304.5411 [gr-qc]

26. R. Aldrovandi, J.G. Pereira, An Introduction to Teleparallel Gravity (Instituto de Fisica Teorica, UNSEP, Sao Paulo). http://www.ift. unesp.br/users/jpereira/tele

27. R. Aldrovandi, J.G. Pereira, K.H. Vu, Selected topics in teleparallel gravity , Braz. J. Phys. 34(4a) (2004). arXiv:gr-qc/0312008
28. J.W. Maluf, The teleparallel equivalent of general relativity. Ann. Phys. 525, 339-357 (2013). arXiv:1303.3897

29. F.W. Hehl, J.D. McCrea, E.W. Mielke, Y. Ne'eman, Metric-affine gauge theory of gravity: field equations, Noether identities, world spinors, and breaking of dilation invariance. Phys. Rep. 258, 1-171 (1995). doi:10.1016/0370-1573(94)00111-F

30. R. Ferraro, F. Fiorini, Modified teleparallel gravity: inflation without inflaton. Phys. Rev. D 75, 084031 (2007). arXiv:gr-qc/0610067

31. T. Harko, F.S.N. Lobo, G. Otalora, E.N. Saridakis, Nonminimal torsion-matter coupling extension of $\mathrm{f}(\mathrm{T})$ gravity. Phys. Rev. D 89, 124036 (2014). arXiv: 1404.6212

32. S. Basilakos, S. Capozziello, M. De Laurentis, A. Paliathanasis, M. Tsamparlis, Noether symmetries and analytical solutions in $\mathrm{f}(\mathrm{T})$ cosmology: a complete study. Phys. Rev. D 88, 103526 (2013). arXiv: 1311.2173

33. K. Bamba, S.D. Odintsov, D. Sez-Gmez, Conformal symmetry and accelerating cosmology in teleparallel gravity. Phys. Rev. D 88, 084042 (2013). arXiv: 1308.5789

34. H.M. Sadjadi, Generalized Noether symmetry in $\mathrm{f}(\mathrm{T})$ gravity. Phys. Lett. B 718, 270-275 (2012). arXiv:1210.0937

35. M.E. Rodrigues, M.J.S. Houndjo, D. Saez-Gomez, F. Rahaman, Anisotropic Universe Models in $\mathrm{f}(\mathrm{T})$ Gravity. Phys. Rev. D 86, 104059 (2012). arXiv: 1209.4859

36. V.F. Cardone, N. Radicella, S. Camera, Accelerating $\mathrm{f}(\mathrm{T})$ gravity models constrained by recent cosmological data. Phys. Rev. D 85, 124007 (2012). arXiv:1204.5294

37. K. Bamba, R. Myrzakulov, S. Nojiri, S.D. Odintsov, Reconstruction of $f(T)$ gravity: Rip cosmology, finite-time future singularities and thermodynamics. Phys. Rev. D 85, 104036 (2012). arXiv: 1202.4057

38. X. Chen, E.N. Saridakis, G. Leon, Phase-space analysis of teleparallel dark energy. JCAP 1207, 005 (2012). arXiv:1202.3781

39. K. Karami, A. Abdolmaleki, Generalized second law of thermodynamics in $\mathrm{f}(\mathrm{T})$-gravity. JCAP 1204, 007 (2012). arXiv:1201.2511

40. H. Wei, X.-J. Guo, L.-F. Wang, Noether symmetry in $\mathrm{f}(\mathrm{T})$ theory. Phys. Lett. B 707, 298-304 (2012). arXiv:1112.2270

41. C.G. Boehmer, T. Harko, F.S.N. Lobo, Wormhole geometries in modified teleparralel gravity and the energy conditions. Phys. Rev. D 85, 044033 (2012). arXiv:1110.5756

42. W. Yi-Peng, C.-Q. Geng, Primordial fluctuations within teleparallelism. Phys. Rev. D 86, 104058 (2012). arXiv:1110.3099

43. S. Capozziello, V.F. Cardone, H. Farajollahi, A. Ravanpak, Cosmography in $\mathrm{f}(\mathrm{T})$-gravity. Phys. Rev. D 84, 043527 (2011). arXiv: 1108.2789

44. C.G. Boehmer, A. Mussa, N. Tamanini, Existence of relativistic stars in $\mathrm{f}(\mathrm{T})$ gravity. Class. Quant. Gravit. 28, 245020 (2011). arXiv: 1107.4455

45. R.-X. Miao, M. Li, Y.-G. Miao, Violation of the first law of black hole thermodynamics in $\mathrm{f}(\mathrm{T})$ gravity. JCAP 1111, 033 (2011). arXiv: 1107.0515

46. X. Meng, Y. Wang, Birkhoff's theorem in the $\mathrm{f}(\mathrm{T})$ gravity. Eur. Phys. J. C 71, 1755 (2011). arXiv:1107.0629

47. H. Wei, X.-P. Ma, H.-Y. Qi, $\mathrm{f}(\mathrm{T})$ theories and varying fine structure constant. Phys. Lett. B 703, 74-80 (2011). arXiv:1106.0102

48. R. Ferraro, F. Fiorini, Non trivial frames for $\mathrm{f}(\mathrm{T})$ theories of gravity and beyond. Phys. Lett. B 702, 75-80 (2011). arXiv:1103.0824

49. Y. Zhang, H. Li, Y. Gong, Z.-H. Zhu, Notes on $\mathrm{f}(\mathrm{T})$ theories. JCAP 1107, 015 (2011). arXiv:1103.0719

50. T. Wang, Static solutions with spherical symmetry in $\mathrm{f}(\mathrm{T})$ theories. Phys. Rev. D 84, 024042 (2011). arXiv: 1102.4410

51. K. Bamba, C.-Q. Geng, C.-C. Lee, L.-W. Luo, Equation of state for dark energy in $\mathrm{f}(\mathrm{T})$ gravity. JCAP 1101, 021 (2011). arXiv: 1011.0508

52. R. Zheng, Q.-G. Huang, Growth factor in $\mathrm{f}(\mathrm{T})$ gravity. JCAP 1103, 002 (2011). arXiv: 1010.3512 
53. J.B. Dent, S. Dutta, E.N. Saridakis, $\mathrm{f}(\mathrm{T})$ gravity mimicking dynamical dark energy. Background and perturbation analysis. JCAP 1101, 009 (2011). arXiv: 1010.2215

54. R.-J. Yang, Conformal transformation in $\mathrm{f}(\mathrm{T})$ theories. Europhys. Lett. 93, 60001 (2011). arXiv:1010.1376

55. K. Karami, A. Abdolmaleki, $\mathrm{f}(\mathrm{T})$ modified teleparallel gravity models as an alternative for holographic and new agegraphic dark energy models. Res. Astron. Astrophys. 13, 757-771 (2013). arXiv: 1009.2459

56. W. Puxun, H.W. Yu, $\mathrm{f}(\mathrm{T})$ models with phantom divide line crossing. Eur. Phys. J. C 71, 1552 (2011). arXiv:1008.3669

57. G.R. Bengochea, Observational information for $\mathrm{f}(\mathrm{T})$ theories and Dark Torsion. Phys. Lett. B 695, 405-411 (2011). arXiv:1008.3188

58. S.-H. Chen, J.B. Dent, S. Dutta, E.N. Saridakis, Cosmological perturbations in $\mathrm{f}(\mathrm{T})$ gravity. Phys. Rev. D 83, 023508 (2011). arXiv: 1008.1250

59. R.-J. Yang, New types of $\mathrm{f}(\mathrm{T})$ gravity. Eur. Phys. J. C 71, 179 (2011). arXiv:1007.3571

60. W. Puxun, H.W. Yu, The dynamical behavior of $\mathrm{f}(\mathrm{T})$ theory. Phys. Lett. B 692, 176-179 (2010). arXiv:1007.2348

61. R. Myrzakulov, Accelerating universe from F(T) gravity. Eur. Phys. J. C 71, 1752 (2011). arXiv: 1006.1120

62. G.G.L. Nashed, W. El Hanafy, A Built-in Inflation in the $\mathrm{f}(\mathrm{T})-$ Cosmology, Eur. Phys. J. C 74(10), 3099 (2014). arXiv:1403.0913

63. T.P. Sotiriou, B. Li, J.D. Barrow, Generalizations of teleparallel gravity and local Lorentz symmetry. Phys. Rev. D 83, 104030 (2011). arXiv:1012.4039
64. B. Li, T.P. Sotiriou, J.D. Barrow, f(T) gravity and local Lorentz invariance. Phys. Rev. D 83, 064035 (2011). arXiv:1010.1041

65. M. Li, R.-X. Miao, Y.-G. Miao, Degrees of freedom of $\mathrm{f}(\mathrm{T})$ gravity. JHEP 1107, 108 (2011). arXiv:1105.5934

66. R. Aldrovandi, J.G. Pereira, Teleparallel gravity, an introduction (Springer, New York, 2013)

67. M.H. Daouda, M.E. Rodrigues, M.J.S. Houndjo, Static anisotropic solutions in $\mathrm{f}(\mathrm{t})$ theory. Eur. Phys. J. C 72, 1890 (2012). arXiv:1109.0528 [physics.gen-ph]

68. S. Bahamonde, C.G. Boehmer, M. Wright, Modified teleparallel theories of gravity. arXiv: 1508.05120 [gr-qc]

69. S. Capozziello, A. Stabile, A. Troisi, Spherical symmetry in $f(\bar{R})$ gravity. Class. Quant. Gravit. 25, 085004 (2008). arXiv:0709.0891 [gr-qc]

70. K. Bamba, C.-Q. Geng, C.-C. Lee, Cosmological evolution in exponential gravity. JCAP 08, 021 (2010). arXiv:1005.4574 [astroph.CO]

71. E.L.B. Junior, M.E. Rodrigues, I.G. Salako, M.J.S. Houndjo, Reconstruction, thermodynamics and stability of CDM Model in $\mathrm{f}(\mathrm{T}, \mathrm{T})$ Gravity. Class. Quant. Gravit. arXiv:1501.00621 [gr-qc] (accepted for publication) 